\title{
Integral Closure of Monomial Ideals on Regular Sequences
}

\author{
Karlheinz Kiyek and Jürgen Stückrad
}

\begin{abstract}
It is well known that the integral closure of a monomial ideal in a polynomial ring in a finite number of indeterminates over a field is a monomial ideal, again. Let $R$ be a noetherian ring, and let $\left(x_{1}, \ldots, x_{d}\right)$ be a regular sequence in $R$ which is contained in the Jacobson radical of $R$. An ideal $\mathfrak{a}$ of $R$ is called a monomial ideal with respect to $\left(x_{1}, \ldots, x_{d}\right)$ if it can be generated by monomials $x_{1}^{i_{1}} \cdots x_{d}^{i_{d}}$. If $x_{1} R+\cdots+x_{d} R$ is a radical ideal of $R$, then we show that the integral closure of a monomial ideal of $R$ is monomial, again. This result holds, in particular, for a regular local ring if $\left(x_{1}, \ldots, x_{d}\right)$ is a regular system of parameters of $R$.
\end{abstract}

\section{Introduction}

Let $A$ be a polynomial ring over a field in a finite number of indeterminates. It is well known that the integral closure $\overline{\mathfrak{A}}$ of a monomial ideal $\mathfrak{A}$ of $A$ is a monomial ideal, again: $\overline{\mathfrak{A}}$ is generated by all monomials $m$ with $m^{l} \in \mathfrak{A}^{l}$ for some $l \in \mathbb{N}$ [cf. [12], section 6.6, Example 6.6.1]. While studying a particular class of ideals in two-dimensional regular local rings [ $\mathrm{cf}$. the example at the end of this paper], the following question arose naturally: Let $R$ be a noetherian ring, and let $\left(x_{1}, \ldots, x_{d}\right)$ be a regular sequence in $R$ such that $\mathfrak{q}:=x_{1} R+\cdots+x_{d} R$ is contained in the Jacobson radical of $R$. Let $\mathfrak{a}$ be an ideal of $R$ that is generated by monomials in $x_{1}, \ldots, x_{d}$; such ideals shall be called monomial ideals. Is the integral closure $\overline{\mathfrak{a}}$ of $\mathfrak{a}$ a monomial ideal, again?

In this paper the question is answered in the positive under the assumption that $R / \mathfrak{q}$ is a reduced ring.

2000 Mathematics Subject Classification: Primary 13B22; Secondary 13B25.

Keywords: Regular sequences, monomial ideals, integral closure of monomial ideals. 
In section 2 we collect some useful results on monomial ideals; in particular, we show that the usual ideal-theoretic operations, applied to monomial ideals, lead again to monomial ideals. It is also shown that for a monomial ideal $\mathfrak{a}$ the ideal $\operatorname{gr}(\mathfrak{a})$ in the associated graded $\operatorname{ring} \operatorname{gr}_{\mathfrak{q}}(R)$ which is a polynomial ring over $R / \mathfrak{q}$ is a monomial ideal.

In section 3 we introduce the notion of a monomial representation of an element of $R$ and we show that, if $R$ is complete, every element of $R$ admits a monomial representation. In section 4 we associate with a monomial ideal $\mathfrak{a}$ the ideal $\widetilde{\mathfrak{a}}$ which is generated by all monomials $m$ in $R$ with $m^{l} \in \mathfrak{a}^{l}$ for some $l \in \mathbb{N}$. In section 5 we study monomial ideals in a polynomial ring over a reduced ring, and we show that for a monomial ideal $\mathfrak{A}$ we have $\overline{\mathfrak{A}}=\widetilde{\mathfrak{A}}$ where $\overline{\mathfrak{A}}$ denotes the integral closure of $\mathfrak{A}$. Let $\mathfrak{a}$ be a monomial ideal in $R$. Using the results of section 5 we show in section 6 that $\overline{\mathfrak{a}}=\widetilde{\mathfrak{a}}$ if $R$ is complete and $\mathfrak{q}$ is a prime ideal. As a last step we show that this equality holds also if $R$ is not necessarily complete, and if $R / \mathfrak{q}$ is a reduced ring.

\section{Monomial Ideals}

\subsection{Basic Definitions}

Notation 1 Let $R$ be a ring. A sequence $\mathbf{x}:=\left(x_{1}, \ldots, x_{d}\right)$ in $R$ is called a weak regular sequence in $R$ if

(a) $x_{i}$ is regular for $R /\left(x_{1}, \ldots, x_{i-1}\right)$ [i.e., the image of $x_{i}$ in $R /\left(x_{1}, \ldots, x_{i-1}\right)$ is a non-zero divisor $]$ for every $i \in\{1, \ldots, d\}$,

and it is called a regular sequence in $R$ if, in addition,

(b) $R \neq \mathbf{x} R$.

In the sequel, we consider regular sequences $\mathbf{x}$ in $R$ with the following additional property:

(c) every permutation $\left(x_{\pi(1)}, \ldots, x_{\pi(d)}\right)$ of $\mathbf{x}$ is a regular sequence in $R$.

Then every subsequence of $\mathbf{x}$ satisfies (a)-(c).

If $R$ is noetherian, and if a regular sequence $\mathbf{x}$ in $R$ is contained in the Jacobson radical [i.e., in the intersection of all maximal ideals] of $R$, then (a) implies (c) [ cf. [2], Ch. X, $\S 9$, no. 7, Th. 1 and Cor. 1], and for the ideal $\mathfrak{q}$ generated by $x_{1}, \ldots, x_{d}$ we have $\bigcap \mathfrak{q}^{p}=(0)$ [cf. [3], Ch. III, $\S 3$, no. 3, Prop. 6].

If $\varphi: R \rightarrow S$ is a flat homomorphism of rings, and if $\varphi(\mathbf{x}) S \neq S$, then the sequence $\varphi(\mathbf{x})$ in $S$ satisfies (a)-(c) [cf. [4], Ch. I, Prop. 1.1.1]. 
(1) For every $d$-tuple $\mathbf{i}:=\left(i_{1}, \ldots, i_{d}\right) \in \mathbb{N}_{0}^{d}$ we define $\operatorname{deg}(\mathbf{i}):=i_{1}+\cdots+i_{d}$, the degree of $\mathbf{i}$, and we write

$$
\mathbf{x}^{\mathbf{i}}:=x_{1}^{i_{1}} \cdots x_{d}^{i_{d}}
$$

Since $\mathbf{x}$ is a regular sequence, we have, for $\mathbf{i}, \mathbf{j} \in \mathbb{N}_{0}^{d}, \mathbf{x}^{\mathbf{i}}=\mathbf{x}^{\mathbf{j}}$ iff $\mathbf{i}=\mathbf{j}$.

(2) An element $m \in R$ is called a monomial with respect to $\mathbf{x}$ if there exists $\mathbf{i} \in \mathbb{N}_{0}^{d}$ with $m=\mathbf{x}^{\mathbf{i}} ; \mathbf{i}$ is determined uniquely by $m$. We call $\operatorname{deg}(m):=\operatorname{deg}(\mathbf{i})$ the degree of $m$.

(3) Let $\mathbf{x}^{\mathbf{i}}=x_{1}^{i_{1}} \cdots x_{d}^{i_{d}}$ be a monomial with respect to $\mathbf{x}$. The set

$$
\operatorname{Supp}\left(\mathbf{x}^{\mathbf{i}}\right):=\left\{j \mid j \in\{1, \ldots, d\}, i_{j} \neq 0\right\}
$$

is called the support of $\mathbf{x}^{\mathbf{i}}$.

(4) Let $M(\mathbf{x})$ be the set of all monomials of $R$ with respect to $\mathbf{x}$. Clearly $M(\mathbf{x})$ is a commutative monoid with cancellation law, and deg: $M(\mathbf{x})$ $\rightarrow \mathbb{N}_{0}$ is a surjective homomorphism of monoids.

(5) An ideal $\mathfrak{a}$ of $R$ is called monomial with respect to $\mathbf{x}$ if it is generated by elements in $M(\mathbf{x})$. In particular, the zero ideal and $R$ itself are monomial ideals.

Remark 1 Let $\mathbf{i}=\left(i_{1}, \ldots, i_{d}\right), \mathbf{j}=\left(j_{1}, \ldots, j_{d}\right) \in \mathbb{N}_{0}^{d}$.

(1) If $\mathbf{x}^{\mathbf{i}} \in \mathbf{x}^{\mathbf{j}} R$, then we have $i_{1} \geqslant j_{1}, \ldots, i_{d} \geq j_{d}$ and $\mathbf{x}^{\mathbf{i}}=\mathbf{x}^{\mathbf{j}} \mathbf{x}^{\mathbf{i}-\mathbf{j}}$. In this case we say that $\mathbf{x}^{\mathbf{j}}$ divides $\mathbf{x}^{\mathbf{i}}$, and we write $\mathbf{x}^{\mathbf{j}} \mid \mathbf{x}^{\mathbf{i}}$.

(2) We define

$$
k_{\tau}:=\min \left\{i_{\tau}, j_{\tau}\right\}, l_{\tau}:=\max \left\{i_{\tau}, j_{\tau}\right\} \quad \text { for } \tau \in\{1, \ldots, d\}
$$

and

$$
\mathbf{k}:=\left(k_{1}, \ldots, k_{d}\right), \mathbf{l}:=\left(l_{1}, \ldots, l_{d}\right)
$$

then

$$
\operatorname{gcd}\left(\mathbf{x}^{\mathbf{i}}, \mathbf{x}^{\mathbf{j}}\right):=\mathbf{x}^{\mathbf{k}}, \operatorname{lcm}\left(\mathbf{x}^{\mathbf{i}}, \mathbf{x}^{\mathbf{j}}\right):=\mathbf{x}^{\mathbf{l}}
$$

is the greatest common divisor resp. the least common multiple of $\mathbf{x}^{\mathbf{i}}$ and $\mathbf{x}^{\mathbf{j}}$. In particular, for monomials $m, n$ we have $m R: n R=$ $(\operatorname{lcm}(m, n) / n) R=(m / \operatorname{gcd}(m, n)) R$. 
Notation 2 For the rest of this paper let $R$ be a noetherian ring, and let $\mathbf{x}=\left(x_{1}, \ldots, x_{d}\right)$ be a fixed sequence in $R$ which satisfies (a)-(c) above; all monomials of $R$ are monomials with respect to $\mathbf{x}$, and all monomial ideals of $R$ are monomial ideals with respect to $\mathbf{x}$. The set of all monomials of $R$ shall be denoted by $M$.

Definition 1 Let $U$ be a subset of $\{1, \ldots, d\}$; we define

$$
\mathfrak{q}_{U}:=\sum_{i \in U} x_{i} R, \mathcal{P}_{U}:=\operatorname{Ass}\left(R / \mathfrak{q}_{U}\right)
$$

If $U=\{1, \ldots, d\}$, then we write

$$
\mathfrak{q}:=\mathfrak{q}_{U}=\sum_{i=1}^{d} x_{i} R, \mathcal{P}:=\operatorname{Ass}(R / \mathfrak{q})
$$

Remark 2 (1) Note that $\operatorname{Ass}(R)=\mathcal{P}_{\emptyset}$.

(2) Let $U \subset\{1, \ldots, d\}, i \in\{1, \ldots, d\} \backslash U$. Then $x_{i}$ is regular for $R / \mathfrak{q}_{U}$, hence, in particular, $x_{i} \notin \mathfrak{p}$ for every $\mathfrak{p} \in \mathcal{P}_{U}$.

Lemma 1 Let $\mathfrak{a}$ be a monomial ideal of $R$, and let $\left\{m_{1}, \ldots, m_{r}\right\}$ be a system of generators of $\mathfrak{a}$ consisting of monomials. Then we have

$$
\operatorname{Ass}(R / \mathfrak{a}) \subset \underset{U \subset \operatorname{Supp}\left(m_{1}\right) \cup \cdots \cup \operatorname{Supp}\left(m_{r}\right)}{\bigcup_{U}} \mathcal{P}_{U}
$$

Proof: There is nothing to prove if $\mathfrak{a}=(0)$. We consider the case that $\mathfrak{a} \neq(0)$. We define $V:=\operatorname{Supp}\left(m_{1}\right) \cup \cdots \cup \operatorname{Supp}\left(m_{r}\right)$. We prove the assertion by induction on $s:=\operatorname{deg}\left(m_{1}\right)+\cdots+\operatorname{deg}\left(m_{r}\right)-r$. If $s=0$, then we have $\mathfrak{a}=\mathfrak{q}_{V}$; in this case the assertion holds. Let $s>0$, and assume that the assertion holds for all monomial ideals of $R$ which admit a system of monomial generators $m_{1}^{\prime}, \ldots, m_{r^{\prime}}^{\prime}$ with $\operatorname{deg}\left(m_{1}^{\prime}\right)+\cdots+\operatorname{deg}\left(m_{r^{\prime}}^{\prime}\right)-r^{\prime}<s$. Now let $\mathfrak{a}$ be a monomial ideal of $R$ having a system of monomial generators $m_{1}, \ldots, m_{r}$ with $\operatorname{deg}\left(m_{1}\right)+\cdots+\operatorname{deg}\left(m_{r}\right)-r=s$. Then there exists $j \in$ $\{1, \ldots, r\}$ with $\operatorname{deg}\left(m_{j}\right) \geq 2$; by relabelling, we may assume that $j=1$.

Let $i \in \operatorname{Supp}\left(m_{1}\right)$; let us label the monomials $m_{1}, \ldots, m_{r}$ in such a way that $i \in \operatorname{Supp}\left(m_{j}\right)$ for $j \in\{1, \ldots, t\}$ and $i \notin \operatorname{Supp}\left(m_{j}\right)$ for $j \in\{t+1, \ldots, r\}$; here we have $t \in\{1, \ldots, r\}$. For $j \in\{1, \ldots, t\}$ we have $m_{j}=x_{i} m_{j}^{\prime}$ where $m_{1}^{\prime}, \ldots, m_{t}^{\prime}$ are monomials. We put

$$
\begin{gathered}
\mathfrak{a}_{1}:=m_{1}^{\prime} R+\cdots+m_{t}^{\prime} R, \mathfrak{a}_{2}=m_{t+1} R+\cdots+m_{r} R, \mathfrak{b}:=\mathfrak{a}_{1}+\mathfrak{a}_{2}, \\
V_{1}:=\bigcup_{j=1}^{t} \operatorname{Supp}\left(m_{j}^{\prime}\right), \quad V_{2}:=\bigcup_{j=t+1}^{r} \operatorname{Supp}\left(m_{j}\right) .
\end{gathered}
$$


If $\mathfrak{a}_{2}=(0)$, then we have $\mathfrak{a}: x_{i}=\mathfrak{b}$. This is also true if $\mathfrak{a}_{2} \neq(0)$. In fact, by our induction assumption we get $\operatorname{Ass}\left(R / \mathfrak{a}_{2}\right) \subset \bigcup_{U \subset V_{2}} \mathcal{P}_{U}$. Using $i \notin V_{2}$, we see that $V_{2} \subset\{1, \ldots, d\} \backslash\{i\}$. From Remark 2 we get the following: If $U \subset V_{2}$, then $x_{i} \notin \mathfrak{p}$ for every prime ideal $\mathfrak{p} \in \mathcal{P}_{U}$, hence $x_{i} \notin \mathfrak{p}$ for every $\mathfrak{p} \in \operatorname{Ass}\left(R / \mathfrak{a}_{2}\right)$, hence $x_{i}$ is regular for $R / \mathfrak{a}_{2}$. This implies that $\mathfrak{a}: x_{i}=\mathfrak{a}_{1}+\mathfrak{a}_{2}=\mathfrak{b}$ since $\mathfrak{a}=x_{i} \mathfrak{a}_{1}+\mathfrak{a}_{2}$.

Therefore the sequence

$$
0 \longrightarrow R / \mathfrak{b} \stackrel{x_{i}}{\longrightarrow} R / \mathfrak{a} \longrightarrow R /\left(\mathfrak{a}+x_{i} R\right) \longrightarrow 0
$$

is exact; note that

$$
\operatorname{Ass}(R / \mathfrak{a}) \subset \operatorname{Ass}(R / \mathfrak{b}) \cup \operatorname{Ass}\left(R /\left(\mathfrak{a}+x_{i} R\right)\right)
$$

We have $\mathfrak{a}+x_{i} R=x_{i} R+m_{t+1} R+\cdots+m_{r} R$. Applying our induction assumption to $\mathfrak{b}$ and to $\mathfrak{a}+x_{i} R$ we obtain

$$
\begin{gathered}
\operatorname{Ass}(R / \mathfrak{b}) \subset \bigcup_{U \subset V_{1} \cup V_{2}} \mathcal{P}_{U} \subset \bigcup_{U \subset V} \mathcal{P}_{U}, \\
\operatorname{Ass}\left(R /\left(\mathfrak{a}+x_{i} R\right)\right) \subset \bigcup_{U \subset\{i\} \cup V_{2}} \mathcal{P}_{U} \subset \bigcup_{U \subset V} \mathcal{P}_{U} .
\end{gathered}
$$

Therefore we get, using $(*)$, that $\operatorname{Ass}(R / \mathfrak{a}) \subset \bigcup_{U \subset V} \mathcal{P}_{U}$.

Corollary 1 If $i \notin \bigcup_{j=1}^{r} \operatorname{Supp}\left(m_{j}\right)$, then we have $\mathfrak{a}: x_{i}=\mathfrak{a}$.

Proof: The element $x_{i}$ is not contained in any of the prime ideals in $\operatorname{Ass}(R / \mathfrak{a})[$ cf. Lemma 1].

\subsection{Operations on Monomial Ideals}

Lemma 2 Let $\mathfrak{a}=m_{1} R+\cdots+m_{r} R$ with $m_{1}, \ldots, m_{r} \in M$ be a monomial ideal in $R$. For every $m \in M$ the ideal $\mathfrak{a}: m$ is monomial, again. More precisely, we have

$$
\mathfrak{a}: m=\sum_{j=1}^{r} \frac{\operatorname{lcm}\left(m_{j}, m\right)}{m} R
$$

Proof: We may assume that $\mathfrak{a} \neq(0)$. We prove the assertion by induction on $\operatorname{deg}(m)$. The case $\operatorname{deg}(m)=0$, i.e., $m=1$, is clear. Let $\operatorname{deg}(m)>0$; then there exists $i \in\{1, \ldots, d\}$ with $x_{i} \mid m$, and we write $m=x_{i} m^{\prime}$ with $m^{\prime} \in M$. As in the proof of Lemma 1 we label the monomials $m_{1}, \ldots, m_{r}$ in such a way that $x_{i} \mid m_{j}$ for $j \in\{1, \ldots, t\}, x_{i} \nmid m_{j}$ for $j \in\{t+1, \ldots, r\}$ 
with $t \in\{0, \ldots, r\}$, and we write, for $j \in\{1, \ldots, t\}, m_{j}=x_{i} m_{j}^{\prime}$ with monomials $m_{1}^{\prime}, \ldots, m_{j}^{\prime}$. Then we have, as above,

$$
\begin{aligned}
\mathfrak{a}: m & =\left(\mathfrak{a}: x_{i}\right): m^{\prime}=\left(\sum_{j=1}^{t} m_{j}^{\prime} R+\sum_{j=t+1}^{r} m_{j} R\right): m^{\prime} \\
& =\sum_{j=1}^{t} \frac{\operatorname{lcm}\left(m_{j}^{\prime}, m^{\prime}\right)}{m^{\prime}} R+\sum_{j=t+1}^{r} \frac{\operatorname{lcm}\left(m_{j}, m^{\prime}\right)}{m^{\prime}} R=\sum_{j=1}^{r} \frac{\operatorname{lcm}\left(m_{j}, m\right)}{m} R .
\end{aligned}
$$

Corollary 2 Let $\mathfrak{a}=m_{1} R+\cdots+m_{r} R$ with $m_{1}, \ldots, m_{r} \in M$ be a monomial ideal in $R$. Let $m \in M$; then we have

$$
\mathfrak{a} \cap m R=\sum_{j=1}^{r} \operatorname{lcm}\left(m_{j}, m\right) R .
$$

Proof: We have $\mathfrak{a} \cap m R=(\mathfrak{a}: m) m$.

Lemma 3 Let $\mathfrak{a}=m_{1} R+\cdots+m_{r} R, \mathfrak{b}=n_{1} R+\cdots+n_{s} R$ with $m_{1}, \ldots, n_{s} \in$ $M$ be monomial ideals in $R$. Then $\mathfrak{a} \cap \mathfrak{b}$ is a monomial ideal; more precisely, we have

$$
\mathfrak{a} \cap \mathfrak{b}=\sum_{i=1}^{r} \sum_{j=1}^{s} \operatorname{lcm}\left(m_{i}, n_{j}\right) R
$$

Proof: It is clear that the right-hand side of $(*)$ is contained in the lefthand side. We prove that the left-hand side of $(*)$ is contained in the right hand side by induction on $s$. For $s=0$ the assertion is clear, and for $s=1$ the assertion follows from Cor. 2. Now we assume that $s \geq 2$, and we define $\mathfrak{b}^{\prime}=n_{1} R+\cdots+n_{s-1} R$. Let $z \in \mathfrak{a} \cap \mathfrak{b}$. We write $z=$ $a_{1} m_{1}+\cdots+a_{r} m_{r}=b_{1} n_{1}+\cdots+b_{s} n_{s}$ with $a_{1}, \ldots, b_{s} \in R$. Since $b_{s} n_{s}=$ $a_{1} m_{1}+\cdots+a_{r} m_{r}-\left(b_{1} n_{1}+\cdots+b_{s-1} n_{s-1}\right)$, we have $b_{s} n_{s} \in\left(\mathfrak{a}+\mathfrak{b}^{\prime}\right) \cap n_{s} R$, hence we can write [cf. Cor. 2]

$$
b_{s} n_{s}=\sum_{i=1}^{r} c_{i} \operatorname{lcm}\left(m_{i}, n_{s}\right)+\sum_{j=1}^{s-1} d_{j} \operatorname{lcm}\left(n_{j}, n_{s}\right) \quad \text { with } c_{1}, \ldots, d_{s-1} \in R .
$$

We define

$$
w:=\sum_{j=1}^{s-1}\left(b_{j} n_{j}+d_{j} \operatorname{lcm}\left(n_{j}, n_{s}\right)\right)
$$


Then we have $w \in \mathfrak{b}^{\prime}$, and since $w=z-\left(c_{1} \operatorname{lcm}\left(m_{1}, n_{s}\right)+\cdots+c_{r} \operatorname{lcm}\left(m_{r}, n_{s}\right)\right) \in \mathfrak{a}$, we have

$$
w \in \mathfrak{a} \cap \mathfrak{b}^{\prime}=\sum_{i=1}^{r} \sum_{j=1}^{s-1} \operatorname{lcm}\left(m_{i}, n_{j}\right) R
$$

by our induction assumption. Then we get

$$
z=w+\sum_{i=1}^{r} c_{i} \operatorname{lcm}\left(m_{i}, n_{s}\right) \in \sum_{i=1}^{r} \sum_{j=1}^{s} \operatorname{lcm}\left(m_{i}, n_{j}\right) R
$$

and therefore the left-hand side of $(*)$ lies in the right hand side.

Collection our results, we have

Proposition 1 Let $\mathfrak{a}, \mathfrak{b}$ be monomial ideals in $R$. Then $\mathfrak{a} \cap \mathfrak{b}, \mathfrak{a} \cdot \mathfrak{b}, \mathfrak{a}: \mathfrak{b}$ are monomial ideals, again. More precisely, if $\mathfrak{a}=m_{1} R+\cdots+m_{r} R$ and $\mathfrak{b}=n_{1} R+\cdots+n_{s} R$ with monomials $m_{1}, \ldots, n_{s} \in M$, then we have

$$
\begin{aligned}
\mathfrak{a} \cap \mathfrak{b} & =\sum_{i=1}^{r} \sum_{j=1}^{s} \operatorname{lcm}\left(m_{i}, n_{j}\right) R \\
\mathfrak{a}: \mathfrak{b} & =\bigcap_{j=1}^{s} \sum_{i=1}^{r} \frac{\operatorname{lcm}\left(m_{i}, n_{j}\right)}{n_{j}} R .
\end{aligned}
$$

If $\mathfrak{c}$ is another monomial ideal, then we have

$$
(\mathfrak{a}+\mathfrak{b}) \cap \mathfrak{c}=(\mathfrak{a} \cap \mathfrak{c})+(\mathfrak{b} \cap \mathfrak{c})
$$

Proof: (2.3) follows from (2.1), and (2.2) is a consequence of Lemma 2 since

$$
\mathfrak{a}: \mathfrak{b}=\bigcap_{j=1}^{s}\left(\mathfrak{a}: n_{j}\right) .
$$

Corollary 3 Let $\mathfrak{a}=m_{1} R+\cdots+m_{r} R$ with $m_{1}, \ldots, m_{r} \in M$ be a monomial ideal in $R$, and let $m \in M$. Then we have $m \in \mathfrak{a}$ iff $m_{i} \mid m$ for some $i \in\{1, \ldots, r\}$.

Proof: We have $m \in \mathfrak{a}$ iff

$$
1 \in \mathfrak{a}: m=\left(\operatorname{lcm}\left(m_{1}, m\right) / m\right) R+\cdots+\left(\operatorname{lcm}\left(m_{r}, m\right) / m\right) R,
$$

hence iff $\operatorname{lcm}\left(m_{i}, m\right) / m=1$ for some $i \in\{1, \ldots, r\}$, and this is the case iff $m_{i} \mid m$ for some $i \in\{1, \ldots, r\}$. 
Corollary 4 Let $\mathfrak{a}$ be a monomial ideal in $R$, and let $m_{1}, \ldots, m_{r}, n_{1}, \ldots, n_{s}$ be monomials with

$$
\mathfrak{a}=\sum_{i=1}^{r} m_{i} R=\sum_{j=1}^{s} n_{j} R .
$$

(1) We assume that $m_{i} \nmid m_{k}$ for all $i, k \in\{1, \ldots, r\}$ with $i \neq k$. Then we have $\left\{m_{1}, \ldots, m_{r}\right\} \subset\left\{n_{1}, \ldots, n_{s}\right\}$.

(2) We assume, furthermore, that $n_{j} \nmid n_{l}$ for all $j, l \in\{1, \ldots, s\}$ with $j \neq l$. Then we have $r=s$ and $\left\{m_{1}, \ldots, m_{r}\right\}=\left\{n_{1}, \ldots, n_{s}\right\}$.

Proof: (1) Note that $\#\left\{m_{1}, \ldots, m_{r}\right\}=r$. Let $i \in\{1, \ldots, r\}$. Then, by Cor. 3, there exist $j \in\{1, \ldots, s\}$ and $k \in\{1, \ldots, r\}$ with $m_{i} \mid n_{j}$ and $n_{j} \mid m_{k}$, hence we have $m_{i} \mid m_{k}$. Therefore we have $i=k$ and $m_{i}=n_{j} \in\left\{n_{1}, \ldots, n_{s}\right\}$. This implies that $\left\{m_{1}, \ldots, m_{r}\right\} \subset\left\{n_{1}, \ldots, n_{s}\right\}$.

(2) This follows immediately from (1).

Remark 3 The result of Cor. 4 implies the following: Every monomial ideal of $R$ admits a uniquely determined minimal set of monomial generators where "minimal" can be understood as "minimal with respect to number" or as "irredundant". We denote this number by $\nu(\mathfrak{a})$. But we can even say more:

Corollary 5 Let $\mathfrak{a}$ be a monomial ideal in $R$, let $r:=\nu(\mathfrak{a})$, and let $\left\{m_{1}, \ldots\right.$, $\left.m_{r}\right\} \subset M$ be a minimal set of monomial generators of $\mathfrak{a}$. Then we have

$$
\mu_{R_{\mathfrak{p}}}\left(\mathfrak{a} R_{\mathfrak{p}}\right)=r \quad \text { for all } \mathfrak{p} \in V\left(\left(x_{1}, \ldots, x_{r}\right)\right) .
$$

Moreover, every set of generators which generates $\mathfrak{a}$ contains at least $r$ elements.

(In a local ring $A$ we denote by $\mu_{A}(M)$ the minimal number of generators of a finitely generated $A$-module $M$.)

Proof: The second statement follows from the first one, and the first statement is obtained from Cor. 4 by replacing $R$ by $R_{\mathfrak{p}}$.

\subsection{The Associated Graded Ring}

Remark 4 The associated graded ring

$$
\operatorname{gr}(R):=\operatorname{gr}_{\mathfrak{q}}(R)=\bigoplus_{p \geq 0} \mathfrak{q}^{p} / \mathfrak{q}^{p+1}=R / \mathfrak{q}\left[\bar{x}_{1}, \ldots, \bar{x}_{d}\right]
$$

is a polynomial ring over $R / \mathfrak{q}$ in $\bar{x}_{1}:=x_{1} \bmod \mathfrak{q}^{2}, \ldots, \bar{x}_{d}:=x_{d} \bmod \mathfrak{q}^{2}$ [cf. [2], Ch. X, $\S 9$, no. 7, Th. 1]. Notice that the sequence $\left(\bar{x}_{1}, \ldots, \bar{x}_{d}\right)$ is a sequence in $\operatorname{gr}(R)$ which satisfies (a)-(c) above. 
(1) Let $\bar{M}=\left\{\overline{\mathbf{x}}^{\mathbf{i}}:=\bar{x}_{1}^{i_{1}} \cdots \bar{x}_{d}^{i_{d}} \mid \mathbf{i} \in \mathbb{N}_{0}^{d}\right\}$ be the set of monomials of the polynomial ring $R / \mathfrak{q}\left[\bar{x}_{1}, \ldots, \bar{x}_{d}\right]$; the map $\mathbf{x}^{\mathbf{i}} \mapsto \overline{\mathbf{x}}^{\mathbf{i}}: M \rightarrow \bar{M}$ is an isomorphism of monoids. An ideal $\mathfrak{A}$ of $\operatorname{gr}(R)$ is called a monomial ideal if it can be generated by elements in $\bar{M}$; such an ideal is a homogeneous ideal of the graded ring $\operatorname{gr}(R)$. Every non-zero element $z \in \operatorname{gr}(R)$ has a unique representation $z=\bar{e}_{1} \bar{m}_{1}+\cdots+\bar{e}_{r} \bar{m}_{r}$ with pairwise distinct monomials $\bar{m}_{1}, \ldots, \bar{m}_{r} \in \bar{M}$ and non-zero elements $\bar{e}_{1}, \ldots, \bar{e}_{r} \in R / \mathfrak{q}$; we call this the monomial representation of $z$.

(2) For every $z \in R$ with $z \notin \bigcap \mathfrak{q}^{p}$ we define the order ord( $(z)$ to be the largest integer $p$ with $z \in \mathfrak{q}^{p}$. Let $p:=\operatorname{ord}(z)$; then we define the initial form of $z$ as $\operatorname{In}(z):=z \bmod \mathfrak{q}^{p+1} \in \operatorname{gr}(R)_{p}$; note that $\operatorname{In}(z)$ is a homogeneous nonzero polynomial of degree $p$. In particular, for a monomial $m \in M \operatorname{ord}(m)$ is defined, and we have ord $(m)=\operatorname{deg}(m)$ and $\operatorname{In}(m)=\bar{m}$.

(3) For every ideal $\mathfrak{a}$ of $R$ we define

$$
\operatorname{gr}(\mathfrak{a}):=\bigoplus_{p \geq 0}\left(\mathfrak{a} \cap \mathfrak{q}^{p}+\mathfrak{q}^{p+1}\right) / \mathfrak{q}^{p+1} \subset \operatorname{gr}(R)
$$

$\operatorname{gr}(\mathfrak{a})$ is a homogeneous ideal in $\operatorname{gr}(R)$. If $\mathfrak{b}$ is another ideal in $R$, then we have $\operatorname{gr}(\mathfrak{a}) \operatorname{gr}(\mathfrak{b}) \subset \operatorname{gr}(\mathfrak{a} \mathfrak{b})$.

(4) Let $\mathfrak{a}=m_{1} R+\cdots+m_{r} R$ with $m_{1}, \ldots, m_{r} \in M$ be a monomial ideal in $R$. Then we have $\operatorname{gr}(\mathfrak{a})=\bar{m}_{1} \operatorname{gr}(R)+\cdots+\bar{m}_{r} \operatorname{gr}(R)$, hence, in particular, $\operatorname{gr}(\mathfrak{a})$ is a monomial ideal in $\operatorname{gr}(R)$ [note that, for $p \in \mathbb{N}_{0}, \mathfrak{a} \cap \mathfrak{q}^{p}$ is generated by the elements $m_{i j}:=\operatorname{lcm}\left(m_{i}, n_{j}\right)$ where $n_{j} \in M$ is of degree $p$ by Lemma 3 , and that $m_{i j} \in \mathfrak{q}^{p+1}$ if $\operatorname{deg}\left(m_{i j}\right)>p$ ]. In particular, for monomial ideals $\mathfrak{a}, \mathfrak{b}$ in $R$ we have $\operatorname{gr}(\mathfrak{a} \mathfrak{b})=\operatorname{gr}(\mathfrak{a}) \operatorname{gr}(\mathfrak{b})$ and $\operatorname{gr}\left(\mathfrak{a}^{i}\right)=(\operatorname{gr}(\mathfrak{a}))^{i}$ for every $i \in \mathbb{N}$.

Remark 5 Now we assume that $\mathfrak{q}$ is a prime ideal of $R$ which is contained in the Jacobson radical of $R$ and we equip $R$ with the $\mathfrak{q}$-adic topology. Then $\bigcap \mathfrak{q}^{p}=(0)$ [cf. [3], Ch. III, $\S 3$, no. 3, Prop. 6], $\operatorname{gr}(R)$ is a domain, hence $R$ is a domain, also, and the order function is a valuation of the quotient field of $R$ [cf. [13], vol. II, Ch. VIII, $\S 1$, Th. 1]. Moreover, all the ideals $\mathfrak{q}_{U}$ for every $U \subset\{1, \ldots, d\}$ are prime ideals as is easily seen by considering the sequence $\left(x_{i} \bmod \mathfrak{q}_{U}\right)_{i \in\{1, \ldots, d\} \backslash U}$ in $R / \mathfrak{q}_{U}$. Therefore all the associated ideals of a monomial ideal $\mathfrak{a}$ of $R$ are of the form $\mathfrak{q}_{U}$ for some $U \subset\{1, \ldots, d\}$ [cf. Lemma 1], and therefore, by considering a primary representation of $\mathfrak{a}$, we get: if $e m \in \mathfrak{a}$ with $e \in R \backslash \mathfrak{q}$ and $m \in M$, then we have $m \in \mathfrak{a}$.

Let $\hat{R}$ be the $\mathfrak{q}$-adic completion of $R$. Then $\mathbf{x}$ is a sequence in $\hat{R}$ which satisfies (a)-(c), $\widehat{\mathfrak{q}}=\mathfrak{q} \hat{R}$ is a prime ideal in $\hat{R}$, and $\hat{R}$ is a faithfully flat $R$-module [cf. [3], Ch. III, $\S 3$, no. 3, Prop. 6]. 


\section{Monomial Representations}

Assumption 1 In this section we assume that $\mathfrak{q}$ is a prime ideal of $R$ which is contained in the Jacobson radical of $R$.

Notation 3 Let $w \in R$ be different from 0 . Then $\operatorname{In}(w) \in \operatorname{gr}(R)$ is a homogeneous polynomial of degree ord $(w)$; therefore there exist uniquely determined and pairwise distinct monomials $m_{1}, \ldots, m_{r} \in M$ having degree $\operatorname{ord}(w)$ and elements $e_{1}, \ldots, e_{r} \in R \backslash \mathfrak{q} \operatorname{such}$ that $\operatorname{In}(w)=\operatorname{In}\left(e_{1} m_{1}+\cdots+\right.$ $\left.e_{r} m_{r}\right)$; we define the set of terms of $w$ by

$$
\operatorname{Tm}(w):=\left\{m_{1}, \ldots, m_{r}\right\}
$$

For $w=0$ we put $\operatorname{In}(w)=0$ and $\operatorname{Tm}(w)=\varnothing$.

Definition 2 We say that $w \in R, w \neq 0$, admits a monomial representation (with respect to $\mathbf{x}$ ), if there exist monomials $m_{1}, \ldots, m_{r} \in M$ and elements $e_{1}, \ldots, e_{r} \in R \backslash \mathfrak{q}$ such that

$$
w=e_{1} m_{1}+\cdots+e_{r} m_{r} \text { and } \nu\left(m_{1} R+\cdots+m_{r} R\right)=r .
$$

In $(*)$ we have $m_{i} \nmid m_{j}$ for all $i, j \in\{1, \ldots, r\}$ with $i \neq j$; in particular, the monomials $m_{1}, \ldots, m_{r}$ are pairwise distinct. For every nonempty subset $U \subset\{1, \ldots, r\}$ clearly $\sum_{i \in U} e_{i} m_{i}=: z$ is a monomial representation of $z$.

Lemma 4 Let $w \in R \backslash\{0\}$. If $w$ admits a monomial representation $w=$ $e_{1} m_{1}+\cdots+e_{r} m_{r}$, then we have

$$
\begin{aligned}
\operatorname{In}(w) & =\sum_{\substack{i=1 \\
\operatorname{deg}\left(m_{i}\right)=\operatorname{ord}(w)}}^{r} \operatorname{In}\left(e_{i}\right) \operatorname{In}\left(m_{i}\right), \\
\operatorname{ord}(w) & =\min \left\{\operatorname{deg}\left(m_{i}\right) \mid i \in\{1, \ldots, r\}\right\}, \\
\operatorname{Tm}(w) & =\left\{m_{i} \mid i \in\{1, \ldots, r\}, \operatorname{deg}\left(m_{i}\right)=\operatorname{ord}(w)\right\} .
\end{aligned}
$$

Proof: Let $s:=\min \left\{\operatorname{deg}\left(m_{i}\right) \mid i \in\{1, \ldots, r\}\right\}$. Then

$$
\operatorname{In}\left(\sum_{\substack{i=1 \\ \operatorname{deg}\left(m_{i}\right)=s}}^{r} e_{i} m_{i}\right)=\sum_{\substack{i=1 \\ \operatorname{deg}\left(m_{i}\right)=s}}^{r} \operatorname{In}\left(e_{i}\right) \operatorname{In}\left(m_{i}\right),
$$

and since $\operatorname{In}\left(e_{i}\right) \neq 0$ for $i \in\{1, \ldots, r\}$, we obtain

$$
\operatorname{ord}\left(\sum_{\substack{i=1 \\ \operatorname{deg}\left(m_{i}\right)=s}}^{r} e_{i} m_{i}\right)=s,
$$


hence $\operatorname{ord}(w)=s$. Clearly we have

$$
\operatorname{In}\left(\sum_{i=1}^{r} e_{i} m_{i}\right)=\operatorname{In}\left(\sum_{\substack{i=1 \\ \operatorname{deg}\left(m_{i}\right)=s}}^{r} e_{i} m_{i}\right)=\operatorname{In}(w) .
$$

Proposition 2 Let $R$ be complete with respect to the $\mathfrak{q}$-adic topology. Every $w \in R, w \neq 0$, admits a monomial representation.

Proof: (1) Let $w \in R, w \neq 0$. Let $\operatorname{Tm}(w)=\left\{m_{1}, \ldots, m_{r}\right\}$. There exist elements $e_{1}, \ldots, e_{r} \in R \backslash \mathfrak{q}$ such that

$$
\operatorname{In}(w)=\operatorname{In}\left(e_{1} m_{1}+\cdots+e_{r} m_{r}\right)
$$

let us put $\iota(w):=e_{1} m_{1}+\cdots+e_{r} m_{r}$. Then we have ord $(w)=\operatorname{ord}(\iota(w))$ and $\operatorname{ord}(w-\iota(w))>\operatorname{ord}(w)$. If $w=0$, then we put $\iota(w)=0$.

(2) Let $w \in R, w \neq 0$. We define a sequence $\left(w_{p}\right)_{p \in \mathbb{N}_{0}}$ in $R$ : Let $w_{0}:=w$; if $p \in \mathbb{N}_{0}$, and if $w_{p}$ is defined, then we define $w_{p+1}:=w_{p}-\iota\left(w_{p}\right)$.

Note the following: If $w_{p}=0$ for one $p \in \mathbb{N}_{0}$, then $w_{q}=0$ for every $q \in \mathbb{N}_{0}$ with $q \geq p$, and if $w_{p} \neq 0$ for one $p \in \mathbb{N}_{0}$, then the elements $w_{0}, \ldots, w_{p-1}$ are different from 0 , and we have

$$
\operatorname{ord}(w)=\operatorname{ord}\left(w_{0}\right)<\operatorname{ord}\left(w_{1}\right)<\cdots<\operatorname{ord}\left(w_{p}\right)
$$

in particular, we have ord $\left(w_{p}\right) \geq p$.

For every $p \in \mathbb{N}_{0}$ let $\mathfrak{a}_{p}$ be that monomial ideal of $R$ which is generated by the monomials in $\operatorname{Tm}\left(w_{0}\right), \ldots, \operatorname{Tm}\left(w_{p}\right)$. Then $\left(\mathfrak{a}_{p}\right)_{p \in \mathbb{N}_{0}}$ is an increasing sequence of ideals in $R$, and therefore it becomes stationary, i.e., there exists $q \in \mathbb{N}_{0}$ with $\mathfrak{a}_{q}=\mathfrak{a}_{q+1}=\cdots=: \mathfrak{a}$. We can write $\mathfrak{a}=m_{1} R+\cdots+m_{r} R$ where $m_{1}, \ldots, m_{r} \in M$ and $r:=\nu(\mathfrak{a})$.

(3) We have

$$
w=w_{p+1}+\sum_{j=0}^{p} \iota\left(w_{j}\right) \quad \text { for every } p \in \mathbb{N}_{0} ;
$$

note that $w_{p+1}=0$ or $\operatorname{ord}\left(w_{p+1}\right) \geq p+1$, hence $w_{p+1} \in \mathfrak{q}^{p+1}$.

Let $j \in \mathbb{N}_{0}$ with $w_{j} \neq 0$. Then we can write $\iota\left(w_{j}\right)$ as a sum

$$
\iota\left(w_{j}\right)=\sum_{i=1}^{r} a_{j i} m_{i}
$$


where the elements $a_{j i} \in R$ for $i \in\{1, \ldots, r\}$ satisfy the following condition: If $\operatorname{ord}\left(w_{j}\right)<\operatorname{deg}\left(m_{i}\right)$, then $a_{j i}=0$, and if $\operatorname{ord}\left(w_{j}\right) \geq \operatorname{deg}\left(m_{i}\right)$ and $a_{j i} \neq 0$, then $a_{j i}$ is a linear combination of monomials of degree ord $\left(w_{j}\right)-\operatorname{deg}\left(m_{i}\right)$ with coefficients which lie in $R \backslash \mathfrak{q}$ [note that the monomials in $\operatorname{Tm}\left(w_{j}\right)$ lie in $\mathfrak{a}]$. For $p \in \mathbb{N}_{0}$ we have

$$
\sum_{j=0}^{p} \iota\left(w_{j}\right)=\sum_{i=1}^{r} e_{p i} m_{i}
$$

with

$$
e_{p i}:=\sum_{j=0}^{p} a_{j i} \quad \text { for every } i \in\{1, \ldots, r\} .
$$

Let $i \in\{1, \ldots, r\}$. There exists a unique $j_{i} \in\{0, \ldots, q\}$ with $\operatorname{ord}\left(w_{j_{i}}\right)=$ $\operatorname{deg}\left(m_{i}\right)$ [cf. (2) and note that $\left\{m_{1}, \ldots, m_{r}\right\}$ is a minimal system of generators of $\mathfrak{a}]$.

We consider any integer $p \geq q$. Then we have $a_{j i}=0$ for $j \in\left\{0, \ldots, j_{i}-\right.$ $1\}, a_{j_{i} i} \in R \backslash \mathfrak{q}$, and $a_{j i} \in \mathfrak{q}^{j-\operatorname{deg}\left(m_{i}\right)}$ for $j \in\left\{j_{i}+1, \ldots, p\right\}$. In particular, $e_{p i} \in R \backslash \mathfrak{q}$. Furthermore, we have

$$
e_{p+1, i}-e_{p i}=a_{p+1, i} \in \mathfrak{q}^{p+1-\operatorname{deg}\left(m_{i}\right)} ;
$$

therefore, the sequence $\left(e_{p i}\right)_{p \geq 0}$ is a Cauchy sequence in $R \backslash \mathfrak{q}$. Since $\mathfrak{q}$ is an open ideal in the $\mathfrak{q}$-adic topology, we have

$$
e_{i}:=\lim _{p \rightarrow \infty} e_{p i} \in R \backslash \mathfrak{q} .
$$

From

$$
\begin{aligned}
\sum_{i=1}^{r} e_{i} m_{i} & =\sum_{i=1}^{r}\left(\lim _{p \rightarrow \infty} e_{p i}\right) m_{i}=\lim _{p \rightarrow \infty}\left(\sum_{i=1}^{r} e_{p i} m_{i}\right) \\
& =\lim _{p \rightarrow \infty}\left(\sum_{j=0}^{p} \iota\left(w_{j}\right)\right)=\lim _{p \rightarrow \infty}\left(w-w_{p+1}\right)
\end{aligned}
$$

and $w_{p+1} \in \mathfrak{q}^{p+1}$ for every $p \in \mathbb{N}_{0}$ we obtain

$$
w=\sum_{i=1}^{r} e_{i} m_{i}
$$


Proposition 3 Let $\mathfrak{a} \neq(0)$ be an ideal in $R$. The following statements are equivalent:

(1) $\mathfrak{a}$ is a monomial ideal.

(2) For every $w \in \mathfrak{a}, w \neq 0$, we have $\operatorname{Tm}(w) \subset \mathfrak{a}$.

Now we assume, in addition, that $R$ is complete in the $\mathfrak{q}$-adic topology. Then the following statements are equivalent with (1) and (2):

(3) Every $w \in \mathfrak{a}, w \neq 0$, admits a monomial representation $w=e_{1} m_{1}+$ $\cdots+e_{r} m_{r}$ with $m_{1}, \ldots, m_{r} \in \mathfrak{a}$.

(4) Let $w \in \mathfrak{a}, w \neq 0$, and let $w=e_{1} m_{1}+\cdots+e_{r} m_{r}$ be a monomial representation of $w$, then $m_{1}, \ldots, m_{r} \in \mathfrak{a}$.

Proof: $(1) \Rightarrow(2)$ : Let $w \in \mathfrak{a}, w \neq 0$, and let $\operatorname{Tm}(w)=\left\{m_{1}, \ldots, m_{r}\right\}$; let $s:=\operatorname{ord}(w)$, hence we have $\operatorname{deg}\left(m_{1}\right)=\cdots=\operatorname{deg}\left(m_{r}\right)=s$ [cf. Lemma 4]. There exist elements $e_{1}, \ldots, e_{r} \in R \backslash \mathfrak{q}$ with $\operatorname{ord}\left(w-\left(e_{1} m_{1}+\cdots+e_{r} m_{r}\right)\right)>s$. Let $i \in\{1, \ldots, r\}$, and define

$$
\mathfrak{b}_{i}:=\mathfrak{a}+m_{1} R+\cdots+m_{i-1} R+m_{i+1} R+\cdots+m_{r} R+\mathfrak{q}^{s+1}
$$

$\mathfrak{b}_{i}$ is a monomial ideal of $R$. Note that $e_{i} m_{i} \in \mathfrak{b}_{i}$, and therefore we have $m_{i} \in \mathfrak{b}_{i}\left[\right.$ cf. Remark 5]. For no monomial $m \in \mathfrak{q}^{s+1}$ we have $m \mid m_{i}$ [since $\left.\operatorname{deg}\left(m_{i}\right)=s<\operatorname{deg}(m)\right]$, and we have $m_{j} \nmid m_{i}$ for $j \in\{1, \ldots, r\}, j \neq i$. Therefore, by Cor. 3, there exists a monomial $m \in \mathfrak{a}$ with $m \mid m_{i}$, hence we have $m_{i} \in \mathfrak{a}$, and therefore we have shown that $\operatorname{Tm}(w) \subset \mathfrak{a}$.

$(2) \Rightarrow(1)$ : Suppose that $\mathfrak{a}$ is not a monomial ideal. This means, in particular, that $\mathfrak{a} \neq R$. Let $\mathfrak{a}^{\prime}$ be the monomial ideal which is generated by all the monomials which lie in $\mathfrak{a}$; then we have $\mathfrak{a}^{\prime} \varsubsetneqq \mathfrak{a}$. By assumption we have $\operatorname{Tm}(w) \subset \mathfrak{a}^{\prime}$ for every $w \in \mathfrak{a}, w \neq 0$. The prime ideals in $\operatorname{Ass}\left(R / \mathfrak{a}^{\prime}\right)$ are of the form $\mathfrak{q}_{U}$ for $U \subset\{1, \ldots, d\}$, hence are contained in $\mathfrak{q}$ [cf. Remark 5]. By Krull's intersection theorem [cf. [13], Vol. I, Ch. 4, § 7, Th. 12'] we have $\bigcap_{n \geq 0}\left(\mathfrak{a}^{\prime}+\mathfrak{q}^{n}\right)=\mathfrak{a}^{\prime}$. Therefore there exists $n \in \mathbb{N}_{0}$ with $\mathfrak{a} \subset \mathfrak{a}^{\prime}+\mathfrak{q}^{n}$, $\mathfrak{a} \not \subset \mathfrak{a}^{\prime}+\mathfrak{q}^{n+1}$. We choose $w \in \mathfrak{a}, w \notin \mathfrak{a}^{\prime}+\mathfrak{q}^{n+1}$; we can write $w=w_{1}+z$ with $w_{1} \in \mathfrak{a}^{\prime}, z \in \mathfrak{q}^{n}$ and $z \notin \mathfrak{q}^{n+1}$. This implies that $z=w-w_{1} \in \mathfrak{a}$, $z \neq 0$, and, by assumption, we have $\operatorname{Tm}(z) \subset \mathfrak{a}$, hence $\operatorname{Tm}(z) \subset \mathfrak{a}^{\prime}$. Let $\operatorname{Tm}(z)=\left\{m_{1}, \ldots, m_{r}\right\}$. Then there exist elements $e_{1}, \ldots, e_{r} \in R \backslash \mathfrak{q}$ such that, putting $z_{1}:=e_{1} m_{1}+\cdots+e_{r} m_{r}$, we have $z_{1} \in \mathfrak{a}^{\prime}$ and $z-z_{1} \in \mathfrak{q}^{n+1}$. This implies that $w=w_{1}+z=w_{1}+z_{1}+\left(z-z_{1}\right) \in \mathfrak{a}^{\prime}+\mathfrak{q}^{n+1}$, in contradiction with the choice of $w$.

Now we assume that $R$ is complete; then every $w \in R, w \neq 0$, admits a monomial representation [cf. Prop. 2]. 
$(2) \Rightarrow(4)$ : Let $w \in \mathfrak{a}, w \neq 0$, and let $w=e_{1} m_{1}+\cdots+e_{r} m_{r}$ be a monomial representation of $w$. We show by induction on $r$ that $\left\{m_{1}, \ldots, m_{r}\right\} \subset \mathfrak{a}$. Let $r=1$, hence $\operatorname{Tm}(w)=\left\{m_{1}\right\} \subset \mathfrak{a}$. Now let $r>1$. It is clear that $\operatorname{Tm}(w) \subset\left\{m_{1}, \ldots, m_{r}\right\}$. We label the elements $m_{1}, \ldots, m_{r}$ in such a way that $\operatorname{Tm}(w)=\left\{m_{1}, \ldots, m_{q}\right\}$ with $q \leq r$. We put $w_{1}:=e_{1} m_{1}+\cdots+e_{q} m_{q}$. Now we have $w_{1} \in \mathfrak{a}$ by assumption. If $q=r$, then the elements $m_{1}, \ldots, m_{q}$ lie in $\mathfrak{a}$. If $q<r$, then we have $w-w_{1}=e_{q+1} m_{q+1}+\cdots+e_{r} m_{r}$, and since $w-w_{1} \in \mathfrak{a}$, we get by our induction assumption that $m_{q+1}, \ldots, m_{r} \in \mathfrak{a}$.

$(4) \Rightarrow(3)$ and $(3) \Rightarrow(1)$ are trivial.

\section{Integral Elements}

Remark 6 Let $S$ be a ring, and let $\mathfrak{a}$ be an ideal in $S$. The integral closure of the Rees ring

$$
\mathcal{R}(\mathfrak{a}, S)=\bigoplus_{p \geq 0} \mathfrak{a}^{p} T^{p} \subset S[T]
$$

in the polynomial ring $S[T]$ is the graded ring $\bigoplus_{p>0} \overline{\mathfrak{a}^{p}} T^{p}$ where, for every $p \in \mathbb{N}, \overline{\mathfrak{a}}^{p}$ is the integral closure of $\mathfrak{a}^{p}$ in $S$ [cf. [10], Ch. II, $\S 5$ ]. In particular, an element $z \in S$ is integral over $\mathfrak{a}$ iff $z T \in S[T]$ is integral over $\bigoplus_{p \geq 0} \mathfrak{a}^{p} T^{p}$.

Notation 4 Let $\mathfrak{a}, \mathfrak{b}$ be monomial ideals in $R$.

(1) We define

$$
\widetilde{\mathfrak{a}}:=\left(\left\{m \in M \mid \text { there exists } l \in \mathbb{N} \text { with } m^{l} \in \mathfrak{a}^{l}\right\}\right)
$$

$\widetilde{\mathfrak{a}}$ is a monomial ideal of $R$. Since the monomials which generate $\widetilde{\mathfrak{a}}$ are integral over $\mathfrak{a}, \widetilde{\mathfrak{a}}$ is an ideal which is integral over $\mathfrak{a}$, and therefore $\widetilde{\mathfrak{a}}$ is contained in the integral closure $\overline{\mathfrak{a}}$ of $\mathfrak{a}$ in $R$, and we have

$$
\mathfrak{a} \subset \widetilde{\mathfrak{a}} \subset \overline{\mathfrak{a}} .
$$

It is clear that $\widetilde{\mathfrak{a}} \widetilde{\mathfrak{b}} \subset \widetilde{\mathfrak{a} \mathfrak{b}}$, and if $\mathfrak{a} \subset \mathfrak{b}$, then we have $\widetilde{\mathfrak{a}} \subset \widetilde{\mathfrak{b}}$.

(2) We show that

$$
\widetilde{\widetilde{a}}=\widetilde{\mathfrak{a}} .
$$

In fact, let $\widetilde{\mathfrak{a}}=m_{1} R+\cdots+m_{r} R$. For every $i \in\{1, \ldots, r\}$ there exists $l_{i} \in \mathbb{N}$ with $m_{i}^{l_{i}} \in \mathfrak{a}^{l_{i}}$. Let $m$ be a monomial in $\widetilde{\widetilde{a}}$. Then there exists $l \in \mathbb{N}$ with $m^{l} \in \widetilde{\mathfrak{a}}^{l}$. This implies that there exist $\left(i_{1}, \ldots, i_{r}\right) \in \mathbb{N}_{0}^{r}$ with $i_{1}+\cdots+i_{r}=l$ and such that $m_{1}^{i_{1}} \cdots m_{r}^{i_{r}}$ divides $m^{l}$ [cf. Cor. 3]. Since $\left(m_{1}^{i_{1}} \cdots m_{r}^{i_{r}}\right)^{l_{1} \cdots l_{r}}$ lies in $\mathfrak{a}^{l l_{1} \cdots l_{r}}$, we see that $m^{l l_{1} \cdots l_{r}}$ lies in $\mathfrak{a}^{l l_{1} \cdots l_{r}}$, also, and this means that $m \in \widetilde{\mathfrak{a}}$. 
(3) By (1) we get $\widetilde{\mathfrak{a}^{p}} \widetilde{\mathfrak{a}}^{q} \subset \widetilde{\mathfrak{a}^{p+q}}$ for all $p, q \in \mathbb{N}_{0}$. Therefore

$$
\widetilde{\mathcal{R}(\mathfrak{a}, R)}:=\bigoplus_{p \geq 0} \widetilde{\mathfrak{a}^{p}} T^{p} \subset R[T]
$$

is a graded $R$-algebra and a graded $R$-subalgebra of $R[T]$, and it contains the Rees ring $\mathcal{R}(\mathfrak{a}, R):=\bigoplus_{p>0} \mathfrak{a}^{p} T^{p}$ of $\mathfrak{a}$ as a graded $R$-subalgebra.

(4) Since $\widetilde{\mathfrak{a}^{p}} \subset \overline{\mathfrak{a}^{p}}$ for every $p \in \mathbb{N}$, the integral closure of $\widetilde{\mathcal{R}(\mathfrak{a}, R)}$ in $R[T]$ is the ring $\bigoplus_{p \geq 0} \overline{\mathfrak{a}^{p}} T^{p}[$ cf. Remark 6].

(5) Just as in [8], Prop. 4.6, one may prove, using (4): For $z \in R$ we have $z \in \overline{\mathfrak{a}}$ iff there exist $p \in \mathbb{N}$ and elements $a_{i} \in \widetilde{\mathfrak{a}^{i}}, i \in\{1, \ldots, p\}$, such that

$$
z^{p}+a_{1} z^{p-1}+\cdots+a_{p}=0 .
$$

Assumption 2 For the rest of this section we again assume that $\mathfrak{q}$ is a prime ideal of $R$ which is contained in the Jacobson radical of $R$. The $\mathfrak{q}$-adic completion of $R$ shall be denoted by $\hat{R}$.

Proposition 4 Let $\mathfrak{a}$ be a monomial ideal of $R$, and let $m=x_{1}^{j_{1}} \cdots x_{d}^{j_{d}} \in M$. The following statements are equivalent:

(1) $m$ is integral over $\mathfrak{a}$.

(2) $m$ is integral over $\mathfrak{a} \hat{R}$.

(3) There exists $l \in \mathbb{N}$ with $m^{l} \in \mathfrak{a}^{l}$.

(4) $\left(j_{1}, \ldots, j_{d}\right)$ lies in the convex hull of $\Gamma+\mathbb{R}_{\geq 0}^{d}$ where $\Gamma \subset \mathbb{N}_{0}^{d}$ is the set of exponents of monomials appearing in $\mathfrak{a}$.

In particular, every monomial in $\overline{\mathfrak{a}}$ lies in $\widetilde{\mathfrak{a}}$.

Proof: $(1) \Rightarrow(2)$ and $(3) \Rightarrow(1)$ hold trivially.

$(2) \Rightarrow(3):$ Let $T^{p}+a_{1} T^{p-1}+\cdots+a_{p} \in \hat{R}[T]$ with $a_{i} \in(\mathfrak{a} \hat{R})^{i}=\mathfrak{a}^{i} \hat{R}$ for $i \in\{1, \ldots, p\}$ be an equation of integral dependence for $m$ over $\mathfrak{a} \hat{R}$. Let $i \in\{1, \ldots, p\}$. Since $\mathfrak{a}^{i}$ is a monomial ideal of $R$, the ideal $\mathfrak{a}^{i} \hat{R}$ is a monomial ideal of $\hat{R}$, and, by Prop. 2, there exist elements $e_{i 1}, \ldots, e_{i r_{i}} \in \hat{R} \backslash \mathfrak{q} \hat{R}$ and monomials $m_{i 1}, \ldots, m_{i r_{i}} \in M$ with

$$
a_{i}=\sum_{j=1}^{r_{i}} e_{i j} m_{i j} .
$$

From Prop. 3 we obtain $m_{i j} \in \mathfrak{a}^{i} \hat{R} \cap R=\mathfrak{a}^{i}$ for $i \in\{1, \ldots, p\}, j \in\left\{1, \ldots, r_{i}\right\}$ [note that $\hat{R}$ is a faithfully flat extension of $R$ ]. Therefore the monomial $m^{p}$ 
lies in the $\hat{R}$-ideal which is generated by the set $\left\{m_{i j} m^{p-i} \mid i \in\{1, \ldots, p\}, j \in\right.$ $\left.\left\{1, \ldots, r_{i}\right\}\right\}$. Using Cor. 3 we find $i \in\{1, \ldots, p\}$ and $j \in\left\{1, \ldots, r_{i}\right\}$ with $m_{i j} m^{p-i} \mid m^{p}$, hence $m_{i j} \mid m^{i}$. Thus, we have shown that $m^{i} \in m_{i j} R \subset \mathfrak{a}^{i}$.

$(3) \Longleftrightarrow(4)$ This is an easy consequence of Cor. 3 and Carathéodory's theorem [for Carathéodory's theorem cf. [11], Th. 17.1].

Corollary 6 Let $\mathfrak{a}$ be a monomial ideal of $R$.

(1) We have $\tilde{\mathfrak{a}} \hat{R}=\widetilde{\mathfrak{a} \hat{R}}$ and $\overline{\mathfrak{a}} \hat{R} \subset \overline{\mathfrak{a} \hat{R}}$.

(2) We have $\widetilde{\operatorname{gr}(\mathfrak{a})}=\operatorname{gr}(\widetilde{\mathfrak{a}})$.

Proof: (1) The first assertion is an easy consequence of Prop. 4, and the second assertion is clear.

(2) Let $\widetilde{\mathfrak{a}}$ be generated by the monomials $m_{1}, \ldots, m_{r}$. Then $\operatorname{gr}(\widetilde{\mathfrak{a}})$ is generated by the monomials $\bar{m}_{1}, \ldots, \bar{m}_{r}$ [cf. (4) in Remark 4]. For every $i \in\{1, \ldots, r\}$ there exists $l_{i} \in \mathbb{N}$ with $m_{i}^{l_{i}} \in \mathfrak{a}^{l_{i}}$, hence $\bar{m}_{i}^{l_{i}} \in \operatorname{gr}\left(\mathfrak{a}^{l_{i}}\right)=\operatorname{gr}(\mathfrak{a})^{l_{i}}$, and therefore we have $\bar{m}_{i} \in \widetilde{\operatorname{gr}(\mathfrak{a})}$. Conversely, let $m \in M$ be a monomial with $\bar{m} \in \widetilde{\operatorname{gr}(\mathfrak{a})}$. Then there exists $l \in \mathbb{N}$ with $\bar{m}^{l} \in(\operatorname{gr}(\mathfrak{a}))^{l}=\operatorname{gr}\left(\mathfrak{a}^{l}\right)$, hence $m^{l} \in \mathfrak{a}^{l}$, and therefore $m \in \widetilde{\mathfrak{a}}$, hence $\bar{m} \in \operatorname{gr}(\widetilde{\mathfrak{a}})$.

\section{Monomial Ideals in Polynomial Rings}

The following result in Prop. 5 should be known, but we could not find a source for it.

Notation 5 Let $(\Gamma, \prec)$ be a totally ordered commutative monoid with neutral element 0 satisfying the following condition:

Every non-empty subset of $\Gamma$ has a smallest element.

This condition is satisfied if $\prec$ is a well-ordering; in particular, a monomial ordering on $\mathbb{N}_{0}^{d}$ satisfies this condition.

Let $R=\bigoplus_{\gamma \in \Gamma} R_{\gamma}$ be a $\Gamma$-graded ring. For $z \in R$ let $z_{\gamma} \in R_{\gamma}$ be the homogeneous component of $z$ of degree $\gamma$, and if $z \neq 0$, then define

$$
\operatorname{Supp}(z):=\left\{\gamma \in \Gamma \mid z_{\gamma} \neq 0\right\}, \operatorname{deg}(z):=\max _{\prec}\{\gamma \mid \gamma \in \operatorname{Supp}(z)\}, z^{*}:=z_{\operatorname{deg}(z)} .
$$

Let $z, w \in R \backslash\{0\}$; then we have $\operatorname{deg}(z w) \preceq \operatorname{deg}(z)+\operatorname{deg}(w)$ if $z w \neq 0$ and $\operatorname{deg}(z+w) \preceq \max _{\prec}\{\operatorname{deg}(z), \operatorname{deg}(w)\}$ if $z+w \neq 0$. Notice that, if $z$ is not homogeneous, then we have $\operatorname{deg}\left(z-z^{*}\right) \prec \operatorname{deg}(z)$. 
Proposition 5 Let $S$ be a $\Gamma$-graded ring, and let $R$ be a $\Gamma$-graded subring of $S$. Then the integral closure $\bar{R}$ of $R$ in $S$ is a $\Gamma$-graded subring of $S$.

Proof: (1) Firstly, we consider the case that every homogeneous element of $S$ which is integral over $R$ already lies in $R$. Then we have to show that $\bar{R}=R$. Suppose that $R \varsubsetneqq \bar{R}$, and choose $z \in \bar{R} \backslash R$ in such a way that $\#(\operatorname{Supp}(z)) \leq \#(\operatorname{Supp}(w))$ for every $w \in \bar{R} \backslash R$. Now $z$ is not homogeneous by our assumption on $R$. If $z^{*} \in \bar{R}$, then we would have $z^{*} \in R$ since $z^{*}$ is homogeneous, hence $z-z^{*} \in \bar{R}$, and therefore $z-z^{*} \in R$ by the choice of $z\left[\right.$ note that $\left.\#\left(\operatorname{Supp}\left(z-z^{*}\right)\right)<\#(\operatorname{Supp}(z))\right]$. Therefore we have $z^{*} \notin \bar{R}$. In particular, we have $\left(z^{*}\right)^{i} \neq 0$ for every $i \in \mathbb{N}$, hence $\left(z^{i}\right)^{*}=\left(z^{*}\right)^{i}$ and $\operatorname{deg}\left(z^{i}\right)=i \operatorname{deg}(z)$ for every $i \in \mathbb{N}$.

Let

$$
\mathcal{V}:=\left\{\mathbf{a}=\left(a_{1}, \ldots, a_{p}\right) \mid a_{1}, \ldots, a_{p} \in R, z^{p}+a_{1} z^{p-1}+\cdots+a_{p}=0\right\} .
$$

Obviously $\mathcal{V}$ is not empty. For every $\mathbf{a}=\left(a_{1}, \ldots, a_{p}\right) \in \mathcal{V}$ we define

$$
\begin{gathered}
\gamma(\mathbf{a}):=\max _{\prec}\left\{\operatorname{deg}\left(a_{i}\right)-i \operatorname{deg}(z) \mid a_{i} \neq 0, i \in\{0,1, \ldots, p\}\right\} \in \Gamma, \\
s(\mathbf{a}):=\min \left\{i \in\{0, \ldots, p\} \mid a_{i} \neq 0, \operatorname{deg}\left(a_{i}\right)-i \operatorname{deg}(z)=\gamma(\mathbf{a})\right\} \in\{0, \ldots, p\}
\end{gathered}
$$

[we define $\left.a_{0}:=1\right]$. Then we have $\gamma(\mathbf{a}) \succeq 0\left[\right.$ since $a_{0}=1 \in R_{0}$ ]. Suppose that there exists $\mathbf{a}=\left(a_{1}, \ldots, a_{p}\right) \in \mathcal{V}$ with $\gamma(\mathbf{a})=0$. Then we have for every $i \in\{1, \ldots, p\}$ with $a_{i} z^{p-i} \neq 0$

$$
\begin{aligned}
\operatorname{deg}\left(a_{i} z^{p-i}\right) & \preceq \operatorname{deg}\left(a_{i}\right)+\operatorname{deg}\left(z^{p-i}\right)=\operatorname{deg}\left(a_{i}\right)+(p-i) \operatorname{deg}(z) \\
& \preceq p \operatorname{deg}(z)+\gamma(\mathbf{a})=p \operatorname{deg}(z) .
\end{aligned}
$$

In $z^{p}+a_{1} z^{p-1}+\cdots+a_{p}=0$ we consider the homogeneous component of degree $p \operatorname{deg}(z)=\operatorname{deg}\left(z^{p}\right)$. Then we get $\left(z^{*}\right)^{p}+a_{1}^{\prime}\left(z^{*}\right)^{p-1}+\cdots+a_{p}^{\prime}=0$ with $a_{i}^{\prime}:=\left\{\begin{array}{lll}a_{i}^{*} & \text { if } a_{i} z^{p-i} \neq 0 \text { and } \operatorname{deg}\left(a_{i} z^{p-i}\right)=p \operatorname{deg}(z), & \text { for } i \in\{1, \ldots, p\} . \\ 0 & \text { else }\end{array}\right.$

But this would imply that $z^{*} \in \bar{R}$, in contradiction with our observation above.

Therefore we have $\gamma(\mathbf{a}) \succ 0$ for every $\mathbf{a} \in \mathcal{V}$. This implies that $s(\mathbf{a})>0$; moreover, we have $s(\mathbf{a}) \leq p-1$ since otherwise $a_{p}^{*}=0$.

Let

$$
\gamma_{0}:=\min _{\prec}\{\gamma(\mathbf{a}) \mid \mathbf{a} \in \mathcal{V}\}, \mathcal{V}_{0}:=\left\{\mathbf{a} \in \mathcal{V} \mid \gamma(\mathbf{a})=\gamma_{0}\right\}
$$


Then we have $\gamma_{0} \succ 0$. We choose $\mathbf{a}=\left(a_{1}, \ldots, a_{p}\right) \in \mathcal{V}_{0}$ with $s(\mathbf{b}) \leq s(\mathbf{a})$ for every $\mathbf{b} \in \mathcal{V}_{0}$. We define

$$
a_{j}^{\prime}:=\left\{\begin{array}{ll}
a_{j}^{*} & \text { if } a_{j} \neq 0, \operatorname{deg}\left(a_{j}\right)-j \operatorname{deg}(z)=\gamma_{0}, \\
0 & \text { else }
\end{array} \text { for } j \in\{1, \ldots, p\} .\right.
$$

By the choice of $s$ we have $a_{1}^{\prime}=\cdots=a_{s-1}^{\prime}=0, a_{s}^{\prime}=a_{s}^{*} \neq 0$, and

$$
a_{s}^{\prime}\left(z^{*}\right)^{p-s}+a_{s+1}^{\prime}\left(z^{*}\right)^{p-s-1}+\cdots+a_{p}^{\prime}=0
$$

[ consider in $z^{p}+a_{1} z^{p-1}+\cdots+a_{p}=0$ the homogeneous component of degree $\left.\gamma_{0}+p \operatorname{deg}(z)\right]$. We multiply $(*)$ by $a_{s}^{\prime p-s-1}$ and obtain

$$
\left(a_{s}^{\prime} z^{*}\right)^{p-s}+a_{s+1}^{\prime}\left(a_{s}^{\prime} z^{*}\right)^{p-s-1}+\cdots+a_{p}^{\prime} a_{s}^{\prime p-s-1}=0 .
$$

Therefore the homogeneous element $a_{s}^{\prime} z^{*}$ is integral over $R$, hence lies in $R$. Since $a_{s}^{\prime} z-a_{s}^{\prime} z^{*}$ is integral over $R$, and since either $a_{s}^{\prime} z=a_{s}^{\prime} z^{*}$ or \# $\left(\operatorname{Supp}\left(a_{s}^{\prime} z-a_{s}^{\prime} z^{*}\right)\right)<\#\left(\operatorname{Supp}\left(a_{s}^{\prime} z\right)\right)$, we have $a_{s}^{\prime} z-a_{s}^{\prime} z^{*} \in R$ by the choice of $z$, hence $a_{s}^{\prime} z \in R$. We define

$$
\bar{a}_{i}:=\left\{\begin{array}{ll}
a_{i} & \text { if } i \neq s, s+1, \\
a_{s}-a_{s}^{\prime} & \text { if } i=s, \\
a_{s+1}+a_{s}^{\prime} z & \text { if } i=s+1
\end{array} \quad \text { for } i \in\{1, \ldots, p\} .\right.
$$

Then we have $\overline{\mathbf{a}}=\left(\bar{a}_{1}, \ldots, \bar{a}_{p}\right) \in R^{p}$, and since $z^{p}+\bar{a}_{1} z^{p-1}+\cdots+\bar{a}_{p}=0$, we have $\overline{\mathbf{a}} \in \mathcal{V}$. We show that we even have $\overline{\mathbf{a}} \in \mathcal{V}_{0}$. We have $\bar{a}_{s}=0$ or $\operatorname{deg}\left(a_{s}-a_{s}^{\prime}\right)-s \operatorname{deg}(z) \prec \operatorname{deg}\left(a_{s}\right)-s \operatorname{deg}(z) \preceq \gamma_{0}$, and we have $\bar{a}_{s+1}=0$ or $\operatorname{deg}\left(a_{s+1}+a_{s}^{\prime} z\right)-(s+1) \operatorname{deg}(z) \preceq \gamma_{0}$, and therefore we have $\gamma(\overline{\mathbf{a}})=\gamma_{0}$. Obviously we have $s(\overline{\mathbf{a}}) \geq s+1$, in contradiction with the choice of $\mathbf{a}$. Therefore we have $\bar{R}=R$.

(2) Now we consider the general case. Let $R^{\prime}:=R[\Sigma]$ where $\Sigma$ is the set of homogeneous elements of $S$ which are integral over $R$; then $R^{\prime}$ is a $\Gamma$-graded subring of $S$. We have $R \subset R^{\prime} \subset \bar{R}$, hence $\bar{R}=\overline{R^{\prime}}$. Since $\overline{R^{\prime}}=R^{\prime}$ by (1), we have $\bar{R}=R^{\prime}$.

Corollary 7 Let $R$ be a $\Gamma$-graded ring, and let $\mathfrak{a}$ be a $\Gamma$-homogeneous ideal of $R$. Then the integral closure of $\mathfrak{a}$ in $R$ is a $\Gamma$-homogeneous ideal of $R$, again.

Proof: We equip the polynomial ring $R[T]$ in a natural way with a $\Gamma \times \mathbb{N}_{0^{-}}$ grading; then we can consider the Rees ring $\mathcal{R}(\mathfrak{a}, R)$ as a $\Gamma \times \mathbb{N}_{0}$-graded 
subring of $R[T]$. The integral closure of $\mathcal{R}(\mathfrak{a}, R)$ in $R[T]$ is a $\Gamma \times \mathbb{N}_{0}$-graded subring by Prop. 5, and $w \in R$ is integral over $\mathfrak{a}$ iff $w T \in R[T]$ lies in

$$
\overline{\mathcal{R}(\mathfrak{a}, R)}=\bigoplus_{p \geq 0} \overline{\mathfrak{a}^{p}} T^{p}
$$

[cf. Remark 6].

Notation 6 For the rest of this section let $k$ be a ring, and let $A=$ $k\left[x_{1}, \ldots, x_{d}\right]$ be the polynomial ring over $k$ in $d$ variables $x_{1}, \ldots, x_{d}$. Then $\left(x_{1}, \ldots, x_{d}\right)$ is a regular sequence in $A$ which satisfies (a)-(c) above; let $M$ be the set of monomials $\mathbf{x}^{\mathbf{i}}=x_{1}^{i_{1}} \cdots x_{d}^{i_{d}}, \mathbf{i} \in \mathbb{N}_{0}^{d}$. Every non-zero $z \in A$ has a unique representation $z=c_{1} m_{1}+\cdots+c_{r} m_{r}$ with non-zero elements $c_{1}, \ldots, c_{r} \in k$ and pairwise distinct monomials $m_{1}, \ldots, m_{r} \in M$; we call this the monomial representation of $z$.

An ideal $\mathfrak{A}$ of $A$ is called a monomial ideal if it is generated by a set of monomials. Let $\mathfrak{A}$ be a monomial ideal in $A$; then $\mathfrak{A}$ is generated by a finite set of monomials [Dickson's Lemma, cf. [1], Ch. 4, Cor. 4.48 and Th. 5.2 or [5], Ch. II, $\S 4$, in particular Exercise 7] and a monomial $m \in M$ belongs to $\mathfrak{A}$ iff it is a multiple of a monomial in $\mathfrak{A}$. Moreover, if $\mathrm{cm} \in \mathfrak{A}$ with $c \in k \backslash\{0\}$ and $m \in M$, then $m \in \mathfrak{A}$.

Corollary 8 Let $\mathfrak{A}$ be a monomial ideal in $A$. Then we have

$$
\overline{\mathfrak{A}}=\operatorname{rad}_{k}(0) A+\widetilde{\mathfrak{A}} .
$$

Proof: Clearly we have $\operatorname{rad}_{k}(0) \subset \overline{\mathfrak{A}}$ and $\widetilde{\mathfrak{A}} \subset \overline{\mathfrak{A}}$. Let $z \in \overline{\mathfrak{A}}, z \neq 0$; since $\overline{\mathfrak{A}}$ is an $\mathbb{N}_{0}^{d}$-homogeneous ideal of $A$ [cf. Cor. 7], there exist $s \in \mathbb{N}$, non-zero elements $c_{1}, \ldots, c_{s} \in k$ and monomials $n_{1}, \ldots, n_{s} \in M$ with $z=$ $c_{1} n_{1}+\cdots+c_{s} n_{s}$ and such that $c_{i} n_{i}$ is integral over $\mathfrak{A}$ for $i \in\{1, \ldots, s\}$. Let $i \in\{1, \ldots, s\}$. Then there exist $p \in \mathbb{N}$, elements $d_{1}, \ldots, d_{p} \in k$ and monomials $m_{1} \in \mathfrak{A}, \ldots, m_{p} \in \mathfrak{A}^{p}$ such that

$$
\left(c_{i} n_{i}\right)^{p}+d_{1} m_{1}\left(c_{i} n_{i}\right)^{p-1}+\cdots+d_{p} m_{p}=0 .
$$

If $d_{1}=\cdots=d_{p}=0$, then we have $c_{i}^{p}=0$, hence $c_{i} \in \operatorname{rad}_{k}(0)$. Otherwise, there exists $l \in\{1, \ldots, p\}$ with $n_{i}^{p}=m_{l} n_{i}^{p-l}$, hence $n_{i}^{l}=m_{l} \in \mathfrak{A}^{l}$, hence $n_{i} \in \widetilde{\mathfrak{A}}$. Therefore we have $z \in \operatorname{rad}_{k}(0) A+\widetilde{\mathfrak{A}}$.

Corollary 9 The following statements are equivalent:

(1) $k$ is a reduced ring.

(2) There exists a monomial ideal $\mathfrak{A}$ in $A$ such that $\overline{\mathfrak{A}}=\widetilde{\mathfrak{A}}$.

(3) For every monomial ideal $\mathfrak{A}$ of $A$ we have $\overline{\mathfrak{A}}=\widetilde{\mathfrak{A}}$. 


\section{The Main Theorem}

We keep the notations and assumptions introduced in section 2 .

Notation 7 (1) A monomial ordering $\prec$ of $\mathbb{N}_{0}^{d}$ is said to be degree-compatible if it satisfies the following condition: for any $\mathbf{i}, \mathbf{j} \in \mathbb{N}_{0}^{d}$ with $\operatorname{deg}(\mathbf{i})<$ $\operatorname{deg}(\mathbf{j})$ we have $\mathbf{i} \prec \mathbf{j}$.

(2) Let $\prec$ be a degree-compatible ordering on $\mathbb{N}_{0}^{d}$. Then every subset of $\mathbb{N}_{0}^{d}$ which is bounded above is finite.

(3) Let $\prec$ be a monomial ordering on $\mathbb{N}_{0}^{d}$. Let $\mathbf{i} \neq \mathbf{j}$ be in $\mathbb{N}_{0}^{d}$. We define $\mathbf{i} \prec_{g} \mathbf{j}$ if $\operatorname{deg}(\mathbf{i})<\operatorname{deg}(\mathbf{j})$ or if $\operatorname{deg}(\mathbf{i})=\operatorname{deg}(\mathbf{j})$ and $\mathbf{i} \prec \mathbf{j}$. Then $\prec_{g}$ is a degree-compatible monomial ordering on $\mathbb{N}_{0}^{d}$.

(4) If $\prec$ is the lexicographical ordering lex on $\mathbb{N}_{0}^{d}$, then $\prec_{g}$ is the degreelexicographical ordering deglex on $\mathbb{N}_{0}^{d}$.

(5) Every monomial ordering $\prec$ on $\mathbb{N}_{0}^{d}$ induces an ordering on $M$ which will be denoted by $\prec$, again.

Proposition 6 We assume that $R / \mathfrak{q}$ is a reduced ring. Let $\mathfrak{a}$ be a monomial ideal of $R$; then $\operatorname{gr}(\widetilde{\mathfrak{a}})$ is the integral closure of the monomial ideal $\operatorname{gr}(\mathfrak{a})$ in $\operatorname{gr}(R)$.

Proof: Since $\widetilde{\mathfrak{a}}$ is integral over $\mathfrak{a}$, obviously $\widetilde{\operatorname{gr}(\mathfrak{a})}=\operatorname{gr}(\widetilde{\mathfrak{a}})[$ cf. Cor. $9(2)]$ is integral over $\operatorname{gr}(\mathfrak{a})$. Let $m \in M$ be a monomial, and assume that $\operatorname{In}(m)=\bar{m}$ is integral over $\operatorname{gr}(\mathfrak{a})$. Then there exists $h \in \mathbb{N}$ with $\operatorname{In}(m)^{h} \in(\operatorname{gr}(\mathfrak{a}))^{h}=$ $\operatorname{gr}\left(\mathfrak{a}^{h}\right)\left[\right.$ cf. Cor. 9], hence we see that $m^{h} \in \mathfrak{a}^{h} \cap \mathfrak{q}^{h \operatorname{deg}(m)} \subset \mathfrak{a}^{h}$, hence $m \in \widetilde{\mathfrak{a}}$, and therefore we obtain that $\operatorname{In}(m) \in \operatorname{gr}(\widetilde{\mathfrak{a}})$.

Remark 7 We assume that $R$ is complete, and that $\mathfrak{q}$ is a prime ideal which is contained in the Jacobson radical of $R$. Let $\prec$ be a degree-compatible monomial ordering on $M$, and let $z \in R \backslash\{0\}$; we define

$$
\operatorname{lm}(z):=\min _{\prec}\{\operatorname{Tm}(z)\} .
$$

Let

$$
z=e_{1} m_{1}+\cdots+e_{r} m_{r}
$$

be a monomial representation of $z$, then we have $\operatorname{lm}(z) \preccurlyeq m_{j}$ for every $j \in$ $\{1, \ldots, r\}$ [cf. Lemma 4 and note that $\prec$ is a degree-compatible ordering], hence we even have

$$
\operatorname{lm}(z)=\min _{\prec}\left\{m_{i} \mid i \in\{1, \ldots, r\}\right\} .
$$

For $z, w \in R \backslash\{0\}$ we obviously have

$$
\operatorname{lm}(z w)=\operatorname{lm}(z) \operatorname{lm}(w) .
$$


Proposition 7 We assume that $R$ is complete, and that $\mathfrak{q}$ is a prime ideal which is contained in the Jacobson radical of $R$. For every monomial ideal $\mathfrak{a}$ of $R$ we have $\overline{\mathfrak{a}}=\tilde{\mathfrak{a}}$.

Proof: (1) We have $\tilde{\mathfrak{a}} \subset \overline{\mathfrak{a}}$ for every monomial ideal $\mathfrak{a}$ of $R$ [cf. (1) in Notation 4]. Suppose that the proposition does not hold. Then the family

$$
\mathcal{I}:=\{\mathfrak{a} \mid \mathfrak{a} \text { monomial ideal of } R, \widetilde{\mathfrak{a}} \varsubsetneqq \overline{\mathfrak{a}}\}
$$

is not empty. For every $\mathfrak{a} \in \mathcal{I}$ we define $r(\mathfrak{a}) \in \mathbb{N}$ in the following way: If $y \in \overline{\mathfrak{a}} \backslash \widetilde{\mathfrak{a}}$, and if $y=e_{1} m_{1}+\cdots+e_{r} m_{r}$ is a monomial representation of $y$ [cf. Prop. 2], then we have $r \geq r(\mathfrak{a})$. Now we choose $\mathfrak{a} \in \mathcal{I}$ in such a way that $r(\mathfrak{a}) \leq r(\mathfrak{b})$ for every $\mathfrak{b} \in \mathcal{I}$. We define $r:=r(\mathfrak{a})$, and we choose $y \in \overline{\mathfrak{a}} \backslash \widetilde{\mathfrak{a}}$ such that $y$ admits a monomial representation $y=e_{1} m_{1}+\cdots+e_{r} m_{r}$ having $r$ terms. By Prop. 4 we have $r \geq 2$. By (5) in Notation 4 there exist $p \in \mathbb{N}$ and $a_{i} \in \widetilde{\mathfrak{a}^{i}}$ for $i \in\{1, \ldots, p\}$ with

$$
y^{p}+a_{1} y^{p-1}+\cdots+a_{p}=0 .
$$

(2) Let $\prec$ be a degree-compatible monomial ordering on $M$. Without loss of generality we may assume that in the monomial representation of $y$ we have $m_{1} \prec m_{2} \prec \cdots \prec m_{r}$, hence that $\operatorname{lm}(y)=m_{1}$, and that $\operatorname{deg}\left(m_{1}\right) \leq$ $\operatorname{deg}\left(m_{2}\right) \leq \cdots \leq \operatorname{deg}\left(m_{r}\right)$. We choose $t \in\{1, \ldots, r\}$ with $\operatorname{deg}\left(m_{1}\right)=$ $\operatorname{deg}\left(m_{2}\right)=\cdots=\operatorname{deg}\left(m_{t}\right)<\operatorname{deg}\left(m_{t+1}\right)$, and we define $y_{1}:=e_{1} m_{1}+\cdots+e_{t} m_{t}$; then we have $\operatorname{In}(y)=\operatorname{In}\left(y_{1}\right)$.

(3) Let

$\mathcal{S}:=\left\{\mathbf{b}=\left(b_{1}, \ldots, b_{p}\right) \mid b_{i} \in \widetilde{\mathfrak{a}^{i}}\right.$ for $\left.i \in\{1, \ldots, p\}, y^{p}+b_{1} y^{p-1}+\cdots+b_{p}=0\right\}$.

The set $\mathcal{S}$ is not empty [cf. (1)]; we define for $\mathbf{b} \in \mathcal{S}$

$$
\begin{gathered}
\rho(\mathbf{b}):=\min _{\prec}\left\{\operatorname{lm}\left(b_{i} y^{p-i}\right) \mid i \in\{1, \ldots, p\}, b_{i} \neq 0\right\} \in M, \\
s(\mathbf{b}):=\min \left\{i \in\{1, \ldots, p\} \mid b_{i} \neq 0, \operatorname{lm}\left(b_{i} y^{p-i}\right)=\rho(\mathbf{b})\right\} \in\{1, \ldots, p\} .
\end{gathered}
$$

(4) There exists $\mathbf{b} \in \mathcal{S}$ with

$$
\rho(\mathbf{b}) \succcurlyeq \operatorname{lm}\left(y^{p}\right)
$$

Proof: Let us suppose, on the contrary, that

$$
\rho(\mathbf{b}) \prec \operatorname{lm}\left(y^{p}\right) \quad \text { for every } \mathbf{b} \in \mathcal{S} \text {. }
$$


This implies that $s(\mathbf{b}) \leq p-1$ for every $\mathbf{b} \in \mathcal{S}$. The set $\{\rho(\mathbf{b}) \mid \mathbf{b} \in \mathcal{S}\}$ is bounded above, hence finite; we define

$$
\rho:=\max _{\prec}\{\rho(\mathbf{b}) \mid \mathbf{b} \in \mathcal{S}\} \in M \text {. }
$$

Furthermore, we define

$$
\mathcal{S}^{\prime}:=\{\mathbf{b} \in \mathcal{S} \mid \rho(\mathbf{b})=\rho\} .
$$

We choose $\mathbf{b}^{\prime}=\left(b_{1}^{\prime}, \ldots, b_{p}^{\prime}\right) \in \mathcal{S}^{\prime}$ in such a way that $s(\mathbf{b}) \leq s\left(\mathbf{b}^{\prime}\right)$ for every $\mathbf{b} \in \mathcal{S}^{\prime}$, and we define $s:=s\left(\mathbf{b}^{\prime}\right)$; note that $1 \leq s \leq p-1$.

Let $i \in\{1, \ldots, p\}$ with $b_{i}^{\prime} \neq 0$. We consider a monomial representation

$$
b_{i}^{\prime}=e_{i 1} m_{i 1}+\cdots+e_{i, r_{i}} m_{i, r_{i}} .
$$

Since $\widetilde{\mathfrak{a}^{i}}$ is a monomial ideal, we have $m_{i 1}, \ldots, m_{i, r_{i}} \in \widetilde{\mathfrak{a}^{i}}[$ cf. Prop. 3]. Without loss of generality we may assume that $m_{i 1} \prec m_{i 2} \prec \cdots \prec m_{i, r_{i}}$. We choose $t_{i} \in\left\{1, \ldots, r_{i}\right\}$ with $\operatorname{deg}\left(m_{i 1}\right)=\cdots=\operatorname{deg}\left(m_{i, t_{i}}\right)<\operatorname{deg}\left(m_{i, t_{i}+1}\right)$, and we define $b_{i}^{\prime \prime}:=e_{i 1} m_{i 1}+\cdots+e_{i, t_{i}} m_{i, t_{i}}$; then we have $\operatorname{In}\left(b_{i}^{\prime}\right)=\operatorname{In}\left(b_{i}^{\prime \prime}\right)$ in $\operatorname{gr}(R)$.

For $i \in\{1, \ldots, p\}$ we define

$$
d_{i}:= \begin{cases}0 & \text { if } b_{i}^{\prime}=0 \text { or if } b_{i}^{\prime} \neq 0 \text { and } \operatorname{lm}\left(b_{i}^{\prime} y^{p-i}\right) \succ \rho \\ b_{i}^{\prime \prime} & \text { if } b_{i}^{\prime} \neq 0 \text { and } \operatorname{lm}\left(b_{i}^{\prime} y^{p-i}\right)=\rho .\end{cases}
$$

Then we have $d_{i} \in \widetilde{\mathfrak{a}^{i}}$ for every $i \in\{1, \ldots, p\}$.

We consider the equation

$$
y^{p}+b_{1}^{\prime} y^{p-1}+\cdots+b_{p}^{\prime}=0 .
$$

For $i \in\{1, \ldots, p\}$ we replace $b_{i}^{\prime}$ by $d_{i}$, and we replace $y$ by $y_{1}$; using the inequality $\rho \prec \operatorname{lm}\left(y^{p}\right)$, we obtain the following equation in $\operatorname{gr}(R)$

$$
\operatorname{In}\left(d_{s}\right) \operatorname{In}\left(y_{1}^{p-s}\right)+\operatorname{In}\left(d_{s+1}\right) \operatorname{In}\left(y_{1}^{p-s-1}\right)+\cdots+\operatorname{In}\left(d_{p}\right)=0 .
$$

We multiply $(* *)$ with $\operatorname{In}\left(d_{s}^{p-s-1}\right)$, and we obtain

$$
\begin{aligned}
\left(\operatorname{In}\left(d_{s} y_{1}\right)\right)^{p-s}+\operatorname{In}\left(d_{s+1}\right)\left(\operatorname{In}\left(d_{s} y_{1}\right)\right)^{p-s-1}+\operatorname{In}\left(d_{s+2} d_{s}\right)\left(\operatorname{In}\left(d_{s} y_{1}\right)\right)^{p-s-2}+ & \\
\cdots+\operatorname{In}\left(d_{p} d_{s}^{p-s-1}\right) & =0 .
\end{aligned}
$$

We have

$$
d_{s+l} d_{s}^{l-1} \in \widetilde{\mathfrak{a}^{s+l}}\left(\widetilde{\mathfrak{a}^{s}}\right)^{l-1} \subset \widetilde{\mathfrak{a}^{(s+1) l}} \text { for } l \in\{1, \ldots, p-s\} .
$$


Therefore we have $\left.\operatorname{In}\left(d_{s+l} d_{s}^{l-1}\right) \in \operatorname{gr}\left(\widetilde{\mathfrak{a}^{(s+1) l}}\right)=\left(\widetilde{\operatorname{gr}\left(\mathfrak{a}^{s+1}\right.}\right)^{l}\right)[$ cf. Cor. 6(2) and (4) in Remark 4] for $l \in\{1, \ldots, p-s\}$, hence $\operatorname{In}\left(d_{s} y_{1}\right)$ is integral over $(\operatorname{gr}(\mathfrak{a}))^{s+1}$ [cf. (5) in Notation 4], $\operatorname{In}\left(m_{s 1} m_{1}\right)$ is integral over $(\operatorname{gr}(\mathfrak{a}))^{s+1}$, also [cf. Cor. 9], and therefore $e_{s 1} e_{1} m_{s 1} m_{1}$ is an element of $\widetilde{\mathfrak{a}^{s+1}}$. We multiply $(*)$ with $\left(e_{s 1} m_{s 1}\right)^{p}$ and we obtain

$$
\left(e_{s 1} m_{s 1} y\right)^{p}+b_{1}^{\prime} e_{s 1} m_{s 1}\left(e_{s 1} m_{s 1} y\right)^{p-1}+\cdots+b_{p}^{\prime}\left(e_{s 1} m_{s 1}\right)^{p}=0 .
$$

Note that

$$
b_{l}^{\prime}\left(e_{s 1} m_{s 1}\right)^{l} \in \widetilde{\mathfrak{a}^{l}}\left(\widetilde{\mathfrak{a}}^{s}\right)^{l} \subset \widetilde{\left(\mathfrak{a}^{s+1}\right)^{l}} \text { for } l \in\{1, \ldots, p\}
$$

and therefore $e_{s 1} m_{s 1} y$ is integral over $\mathfrak{a}^{s+1}\left[\right.$ cf. (5) in Notation 4]. Let $y^{\prime}:=$ $y-e_{1} m_{1}$; then $e_{s 1} m_{s 1} y^{\prime}$ is integral over $\mathfrak{a}^{s+1}$, and $e_{s 1} m_{s 1} y^{\prime}=\sum_{i=2}^{r} e_{i} e_{s 1} m_{s 1} m_{i}$ admits a monomial representation having only $r-1$ terms.

We have $e_{s 1} m_{s 1} y^{\prime} \in \widetilde{\mathfrak{a}^{s+1}}$ [this is clear if $\overline{\mathfrak{a}^{s+1}}=\widetilde{\mathfrak{a}^{s+1}}$, and if $\overline{\mathfrak{a}^{s+1}} \supsetneqq \widetilde{\mathfrak{a}^{s+1}}$, then $\mathfrak{a}^{s+1}$ lies in $\mathcal{I}$, and by the choice of $r\left[\right.$ cf. (1)] we get $e_{s 1} m_{s 1} y^{\prime} \in \widetilde{\mathfrak{a}^{s+1}}$ in this case, also]. Since $e_{s 1} m_{s 1} y^{\prime}$ and $e_{1} e_{s 1} m_{1} m_{s 1}$ lie in $\widetilde{\mathfrak{a}^{s+1}}$, the element $e_{s 1} m_{s 1} y$ lies in $\widetilde{\mathfrak{a}^{s+1}}$, also.

We define [ note that $s \leq p-1$ ]

$$
\widetilde{b}_{i}:=\left\{\begin{array}{ll}
b_{i}^{\prime} & \text { if } i \neq s, s+1, \\
b_{s}^{\prime}-e_{s 1} m_{s 1} & \text { if } i=s, \\
b_{s+1}^{\prime}+e_{s 1} m_{s 1} y & \text { if } i=s+1
\end{array} \quad \text { for } i \in\{1, \ldots, p\}\right.
$$

We have $\mathbf{b}^{\prime} \in \mathcal{S}, e_{s 1} m_{s 1} \in \widetilde{\mathfrak{a}^{s}}$ and $e_{s 1} m_{s 1} y \in \widetilde{\mathfrak{a}^{s+1}}$, hence we have $\widetilde{b}_{i} \in \widetilde{\mathfrak{a}^{i}}$ for $i \in\{1, \ldots, p\}$. Clearly we have

$$
y^{p}+\widetilde{b}_{1} y^{p-1}+\cdots+\widetilde{b}_{p}=0,
$$

and therefore $\widetilde{\mathbf{b}}:=\left(\widetilde{b}_{1}, \ldots, \widetilde{b}_{p}\right)$ lies in $\mathcal{S}$, and this implies that $\rho(\widetilde{\mathbf{b}}) \preccurlyeq \rho$ by the choice of $\rho$. We show that $\widetilde{\mathbf{b}}$ even lies in $\mathcal{S}^{\prime}$.

We have $\widetilde{b}_{s}=0$ or $\widetilde{b}_{s}=e_{s 2} m_{s 2}+\cdots+e_{s, r_{s}} m_{s, r_{s}}$ and $\operatorname{lm}\left(\widetilde{b}_{s}\right)=m_{s 2} \succ$ $m_{s 1}=\operatorname{lm}\left(b_{s}^{\prime}\right)=\rho$. We have $\operatorname{lm}\left(e_{s 1} m_{s 1} y^{p-s}\right)=\rho$, and if $b_{s+1}^{\prime} \neq 0$, then we have $\operatorname{lm}\left(b_{s+1}^{\prime} y^{p-s-1}\right) \succcurlyeq \rho$. Therefore we have $\operatorname{lm}\left(\widetilde{b}_{s+1} y^{p-s-1}\right) \succcurlyeq \rho$, and since $\rho\left(\mathbf{b}^{\prime}\right)=\rho$, we obtain $\rho(\widetilde{\mathbf{b}}) \succcurlyeq \rho$. This implies that $\rho(\widetilde{\mathbf{b}})=\rho$, hence we get, in fact, that $\widetilde{\mathbf{b}} \in \mathcal{S}^{\prime}$.

Now we have $\widetilde{b}_{s}=0$ or $\operatorname{lm}\left(\widetilde{b}_{s}\right) \succ \rho$ and $\widetilde{b}_{i}=b_{i}^{\prime}$ for $i \in\{1, \ldots, s-1\}$, and this implies $s(\widetilde{\mathbf{b}})>s\left(\mathbf{b}^{\prime}\right)=s$, in contradiction with the choice of $\mathbf{b}^{\prime}$. 
(5) By (4) there exists $\mathbf{b} \in \mathcal{S}$ with $\operatorname{lm}\left(b_{i} y^{p-i}\right) \succcurlyeq \operatorname{lm}\left(y^{p}\right)$ for every $i \in$ $\{1, \ldots, p\}$ with $b_{i} \neq 0$.

Let $i \in\{1, \ldots, p\}$ with $b_{i} \neq 0$, and let $b_{i}=e_{i 1} m_{i 1}+\cdots+e_{i, r_{i}} m_{i, r_{i}} \in \widetilde{\mathfrak{a}^{i}}$ be a monomial representation of $b_{i}$; without loss of generality we may assume that $m_{i 1} \prec m_{i 2} \prec \cdots \prec m_{i, r_{i}}$, which implies that $m_{i 1}=\operatorname{lm}\left(b_{i}\right)$. We choose $t_{i} \in\left\{1, \ldots, r_{i}\right\}$ with $\operatorname{deg}\left(m_{i 1}\right)=\cdots=\operatorname{deg}\left(m_{i, t_{i}}\right)<\operatorname{deg}\left(m_{i, t_{i}+1}\right)$, and we define

$$
b_{i}^{\prime}:=e_{i 1} m_{i 1}+\cdots+e_{i, t_{i}} m_{i, t_{i}} ;
$$

note that $\operatorname{In}\left(b_{i}\right)=\operatorname{In}\left(b_{i}^{\prime}\right)$. We have $m_{i j} \in \widetilde{\mathfrak{a}^{i}}$ for $j \in\left\{1, \ldots, r_{i}\right\}$ [cf. Prop. 3], hence, in particular, $b_{i}^{\prime} \in \widetilde{\mathfrak{a}}^{i}$.

Now let $i \in\{1, \ldots, p\}$; we define

$$
c_{i}:= \begin{cases}0 & \text { if } b_{i}=0 \text { or if } b_{i} \neq 0 \text { and } \operatorname{lm}\left(b_{i} y^{p-i}\right) \succ \operatorname{lm}\left(y^{p}\right), \\ b_{i}^{\prime} & \text { if } b_{i} \neq 0 \text { and } \operatorname{lm}\left(b_{i} y^{p-i}\right)=\operatorname{lm}\left(y^{p}\right) .\end{cases}
$$

Clearly we have $c_{i} \in \widetilde{\mathfrak{a}^{i}}$. From $y^{p}+b_{1} y^{p-1}+\cdots+b_{p}=0$ we obtain the following equation in $\operatorname{gr}(R)$

$$
\operatorname{In}\left(y_{1}\right)^{p}+\operatorname{In}\left(c_{1}\right) \operatorname{In}\left(y_{1}\right)^{p-1}+\cdots+\operatorname{In}\left(c_{p}\right)=0 .
$$

Now we have $\operatorname{In}\left(c_{i}\right) \in \operatorname{gr}\left(\widetilde{\mathfrak{a}^{i}}\right)$ for every $i \in\{1, \ldots, p\}$. Just as in (4) we see that $\operatorname{In}\left(y_{1}\right)$ is integral over $\operatorname{gr}(\mathfrak{a})$ and that therefore $\operatorname{In}\left(m_{1}\right)$ is integral over $\operatorname{gr}(\mathfrak{a})$, hence we have $m_{1} \in \widetilde{\mathfrak{a}}$, hence $e_{1} m_{1} \in \widetilde{\mathfrak{a}}$. Now $y^{\prime}:=y-e_{1} m_{1}$ lies in $\overline{\mathfrak{a}}$, and therefore $y^{\prime}$ lies in $\widetilde{\mathfrak{a}}$ by the choice of $r$. From this we get that $y=y^{\prime}+e_{1} m_{1}$ lies in $\widetilde{\mathfrak{a}}$, in contradiction with the choice of $y$.

Theorem 1 Let $R$ be a noetherian ring, let $\mathbf{x}=\left(x_{1}, \ldots, x_{d}\right)$ be a regular sequence in $R$, and assume that $\mathfrak{q}:=\mathbf{x} R$ is contained in the Jacobson radical of $R$ and that $R / \mathfrak{q}$ is a reduced ring. For every monomial ideal $\mathfrak{a}$ of $R$ we have $\overline{\mathfrak{a}}=\widetilde{\mathfrak{a}} ;$ in particular, $\overline{\mathfrak{a}}$ is a monomial ideal, also.

Proof: (1) Firstly, let $\mathfrak{q}$ be a prime ideal. Let $y \in \overline{\mathfrak{a}}$. We have $\overline{\mathfrak{a}} \hat{R} \subset \overline{(\mathfrak{a} \hat{R})}$ and $\widetilde{\mathfrak{a} \hat{R}}=\widetilde{\mathfrak{a}} \hat{R}[$ cf. Cor. 6], hence $y \in \overline{\mathfrak{a} \hat{R}}=\widetilde{\mathfrak{a} \hat{R}}=\widetilde{\mathfrak{a}} \hat{R}$ [cf. Prop. 7], and since $\tilde{\mathfrak{a}} \hat{R} \cap R=\widetilde{\mathfrak{a}}$ we obtain $y \in \widetilde{\mathfrak{a}}$. Thus, we have shown that $\overline{\mathfrak{a}}=\widetilde{\mathfrak{a}}$.

(2) Now we consider the case that $R / \mathfrak{q}$ is reduced.

(a) Let $\mathfrak{p} \in \operatorname{Ass}(R / \mathfrak{q})$. Then $\mathfrak{q} R_{\mathfrak{p}}$ is the maximal ideal of $R_{\mathfrak{p}}$, hence we have $\widetilde{\mathfrak{a} R_{\mathfrak{p}}}=\overline{\mathfrak{a} R_{\mathfrak{p}}}$ by (1). Obviously we have $\widetilde{\mathfrak{a} R_{\mathfrak{p}}}=\widetilde{\mathfrak{a}} R_{\mathfrak{p}}$ and $\overline{\mathfrak{a}} R_{\mathfrak{p}} \subset \overline{\mathfrak{a} R_{\mathfrak{p}}}$. Therefore we have $\overline{\mathfrak{a}} R_{\mathfrak{p}} \subset \widetilde{\mathfrak{a}} R_{\mathfrak{p}}$.

(b) For every $\mathfrak{p} \in \operatorname{Ass}(R / \mathfrak{q})$ there exists, by (a), an element $s_{\mathfrak{p}} \in R \backslash \mathfrak{p}$ with $\overline{\mathfrak{a}} \subset \widetilde{\mathfrak{a}}: s_{\mathfrak{p}}$. Let $\mathfrak{b}$ be the ideal generated by the elements $s_{\mathfrak{p}}$; then we 
have $\overline{\mathfrak{a}} \subset \widetilde{\mathfrak{a}}: \mathfrak{b}$. Let $\mathfrak{p}^{\prime} \in \operatorname{Ass}(R / \widetilde{\mathfrak{a}})$. Since $\widetilde{\mathfrak{a}}$ is a monomial ideal, there exists $U \subset\{1, \ldots, d\}$ with $\mathfrak{p}^{\prime} \in \operatorname{Ass}\left(R / \mathfrak{q}_{U}\right)$ [cf. Lemma 1]. Repeated application of Lemma 1 in [13], vol. II, Appendix 6, shows that there exists a prime ideal $\mathfrak{p} \in \operatorname{Ass}(R / \mathfrak{q})$ with $\mathfrak{p}^{\prime} \subset \mathfrak{p}$. Therefore $\mathfrak{b}$ is not contained in any prime ideal in $\operatorname{Ass}(R / \widetilde{\mathfrak{a}})$, hence $\widetilde{\mathfrak{a}}: \mathfrak{b}=\widetilde{\mathfrak{a}}$, hence $\overline{\mathfrak{a}} \subset \widetilde{\mathfrak{a}}$. The inclusion $\widetilde{\mathfrak{a}} \subset \overline{\mathfrak{a}}$ was noticed in (1) of Notation 4 , and therefore we have $\overline{\mathfrak{a}}=\widetilde{\mathfrak{a}}$.

Example 1 Let $R$ be a regular local two-dimensional ring, and let $\{x, y\}$ be a regular system of parameters of $R$. Let $m>n>1$ be coprime integers, and write $m=s_{1} n+n_{1}$ with $1 \leq n_{1}<n$. Let $\mathfrak{a}$ be the ideal of $R$ generated by $x^{m}$ and $y^{n}$. Then $\mathfrak{a}$ is a monomial ideal. It can be shown [cf. [7]] that the integral closure $\wp$ of $\mathfrak{a}$ has a minimal system of generators $\left\{x^{m-\sigma_{m, n}(i)} y^{i}\right.$ $i \in\{0, \ldots, n\}\}$ where $\sigma_{m, n}:\{0, \ldots, n\} \rightarrow\{0, \ldots, m\}$ is a strictly increasing function; in particular, one has

$$
\sigma_{m, n}(0)=0, \sigma_{m, n}(1)=s_{1}, \sigma_{m, n}(n-1)=m-\left(s_{1}+1\right), \sigma_{m, n}(n)=m,
$$

and

$$
\sigma_{m, n}(i+j) \geq \sigma_{m, n}(i)+\sigma_{m, n}(j) \text { for } i, j \in\{0, \ldots, n\} \text { with } i+j \leq n .
$$

Moreover, the polar ideal $\mathfrak{P}_{\wp}$ of $\wp$ has

$$
\left\{x^{m-\sigma_{m, n}(i+1)} y^{i} \mid i \in\{0, \ldots, n-1\}\right\}
$$

as minimal set of generators.

\section{References}

[1] Becker, Th. and Weispfenning, V.: Gröbner Bases. Graduate Texts in Mathematics 141. Springer, New York, 1993.

[2] Bourbaki, N.: Algèbre. Masson, Paris, 1980.

[3] Bourbaki, N.: Algèbre Commutative. Masson, Paris, 1983.

[4] Bruns, W. And Herzog, J.: Cohen-Macaulay Rings. Cambridge University Press, Cambridge, 1993.

[5] Cox, D., Little, J. And O'Shea, D.: Ideals, Varieties, and Algorithms. An introduction to computational algebraic geometry and commutative algebra. Undergraduate Texts in Mathematics, Springer, New York, 1992.

[6] Fröberg, R.: An Introduction to Gröbner Bases. Wiley, New York, 1997.

[7] Greco, S. And Kiyek, K.: The polar ideal of a simple complete ideal having one characteristic pair. Preprint, Politecnico di Torino, Rapporto interno N. 32. 
[8] Herrmann, M., Ikeda, S. and Orbanz, U.: Equimultiplicity and Blowing up. Springer, Berlin, 1988.

[9] Kempf, G., Knudsen, F., Mumford, D. And Saint-Donat, B.: Toroidal embeddings I. Lecture notes in Mathematics 339: Springer-Verlag, Berlin, 1973.

[10] Lipman, J.: Rational singularities with applications to algebraic surfaces and unique factorization. Inst. Hautes Études Sci. Publ. Math. 36 (1969), 195-279.

[11] Rockafellar, R. T.: Convex Analysis. Princeton Landmarks in Mathematics, Princeton University Press, 10th printing, Princeton, 1997.

[12] Vasconcelos, W.: Computational Methods in Commutative Algebra and Algebraic Geometry. Springer-Verlag, Berlin, 1998.

[13] Zariski, O., And Samuel, P.: Commutative Algebra. The University Series in Higher Mathematics. D. Van Nostrand Co., Inc., Princeton, N. J.Toronto-London-New York, 1960.

Recibido: 20 de febrero de 2002

Revisado: 23 de octubre de 2002

Karlheinz Kiyek Institut für Mathematik Fakultät für Elektrotechnik, Informatik und Mathematik Universität Paderborn 33098 Paderborn, Deutschland karlh@uni-paderborn.de

Jürgen Stückrad Mathematisches Institut Universität Leipzig Augustusplatz 10-11 04109 Leipzig, Deutschland stueckrad@mathematik. uni-leipzig.de 